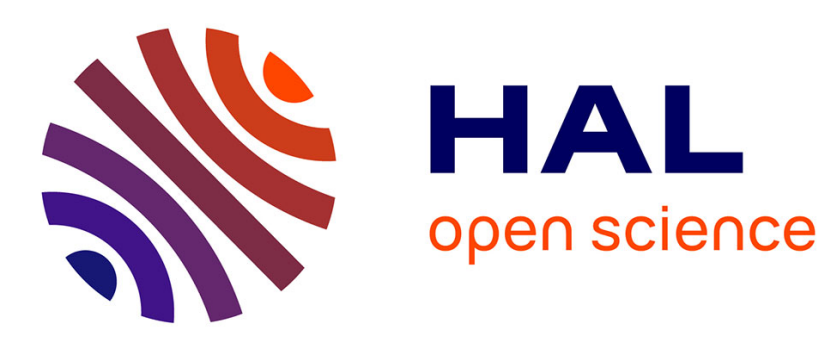

\title{
Three dimensional liquid and vapor distribution in the wick of capillary evaporators
}

\author{
Laetitia Mottet, Typhaine Coquard, Marc Prat
}

\section{To cite this version:}

Laetitia Mottet, Typhaine Coquard, Marc Prat. Three dimensional liquid and vapor distribution in the wick of capillary evaporators. International Journal of Heat and Mass Transfer, 2015, 83, pp.636-651. 10.1016/j.ijheatmasstransfer.2014.12.048 . hal-01331859

\section{HAL Id: hal-01331859 \\ https://hal.science/hal-01331859}

Submitted on 14 Jun 2016

HAL is a multi-disciplinary open access archive for the deposit and dissemination of scientific research documents, whether they are published or not. The documents may come from teaching and research institutions in France or abroad, or from public or private research centers.
L'archive ouverte pluridisciplinaire HAL, est destinée au dépôt et à la diffusion de documents scientifiques de niveau recherche, publiés ou non, émanant des établissements d'enseignement et de recherche français ou étrangers, des laboratoires publics ou privés. 


\section{OATAO}

\section{Open Archive TOULOUSE Archive Ouverte (OATAO)}

OATAO is an open access repository that collects the work of Toulouse researchers and makes it freely available over the web where possible.

This is an author-deposited version published in : http://oatao.univ-toulouse.fr/ Eprints ID : 15923

To link to this article : DOI:10.1016/j.ijheatmasstransfer.2014.12.048 URL : http://dx.doi.org/10.1016/j.ijheatmasstransfer.2014.12.048

To cite this version : Mottet, Laetitia and Coquard, Typhaine and Prat, Marc Three dimensional liquid and vapor distribution in the wick of capillary evaporators. (2015) International Journal of Heat and Mass Transfer, vol. 83. pp. 636-651. ISSN 0017-9310

Any correspondence concerning this service should be sent to the repository administrator: staff-oatao@,listes-diff.inp-toulouse.fr 


\title{
Three dimensional liquid and vapour distribution in the wick of capillary evaporators
}

\author{
L. Mottet ${ }^{\mathrm{a}, \mathrm{b}, \mathrm{c}, \mathrm{d}}$, T. Coquard ${ }^{\mathrm{c}}$, M. Prat ${ }^{\mathrm{a}, \mathrm{b}, *}$ \\ ${ }^{a}$ Université de Toulouse, INPT, UPS, IMFT, Toulouse, France \\ ${ }^{\mathrm{b}} \mathrm{CNRS}$, IMFT, Toulouse, France \\ ${ }^{c}$ Airbus Defence and Space, Toulouse, France \\ ${ }^{\mathrm{d}}$ Centre National d'études spatiales, Toulouse, France
}

Keywords:

Loop heat pipes

Capillary evaporators

Porous wick

Pore-network model

\begin{abstract}
A B S T R A C T
Heat and mass transfer with liquid-vapour phase change in a representative unit cell of a capillary evaporator is studied using a mixed pore network model. The model combines the computation of temperature and pressure fields in vapour and liquid pores according to mean field approaches with pore scale invasion rules depending on the capillary pressure thresholds associated with each local constriction between two pores. The metallic body through which heat is transferred to the porous wick is also taken into account in the simulations. After comparisons with a visualisation experiment, numerical simulations performed in three dimensional pore networks lead to the identification of three main regimes depending on the applied heat load. Compared with previous works using the so-called vapour pocket assumption, the 3D simulations reveal a regime where the phase distribution within the wick is fundamentally different. This regime is characterised by the coexistence of both the liquid and vapour phases underneath the casing within the wick. This regime is shown to correspond to the best evaporator performance.
\end{abstract}

\section{Introduction}

Capillary pumped loops (CPL) and Loop heat pipes (LHPs), e.g. $[1,2]$, are cooling devices developed to meet the increasingly demanding thermal control problems of high-end electronics and radio frequency devices. First developed in relation with aerospace applications, these devices are also studied nowadays for railbound transport, automobile transport or aeronautics applications. A sketch of LHP is shown in Fig. 1a. It consists of an evaporator, a compensation chamber (also called reservoir), vapour and liquid transport lines and a condenser.

Two main types of evaporator are encountered in LHP: flat or cylindrical. Fig. 1b shows a schematic of the cross-section of a cylindrical evaporator. Both types of evaporator, i.e. flat or cylindrical, consist of a liquid-passage core, vapour-evacuation grooves, an outer casing and a capillary porous wick. Heat applied to the outer casing leads to the evaporation of the liquid inside the wick or at the wick/vapour groove interface. The produced vapour is collected in the vapour grooves and flows through the vapour transport line

\footnotetext{
* Corresponding author at: Université de Toulouse, INPT, UPS, IMFT, Toulouse, France.

E-mail address: mprat@imft.fr (M. Prat).
}

towards the condenser. The menisci formed at the wick/grooves interface or inside the wick adjust themselves to establish a capillary suction that balances the total pressure drop in the device. Unwrapping the cylindrical evaporator leads to a structure close to a flat evaporator. In what follows we will concentrate on the unit cell shown in Fig. 2, which is therefore representative of both types of evaporator.

Owing to their technological importance, LHP have been the subject of quite a few investigations. Roughly, one can distinguish two main types of works. The works aiming at studying the whole LHP, e.g. [3-5] to name but a few, and the works focussing on one of the components, the condenser or more often the evaporator because evaporator is generally considered as the key component in this system, e.g. [6,7]. The present work belongs to the second category. We focus on the heat and mass transfer with phase change inside the evaporator. One can then again distinguish two types of works as regards the modelling of heat and mass transfers in the porous wick of evaporator: the works where the wick is assumed to be liquid saturated, e.g. [8-12], and the works where the wick can be partially invaded by the vapour. The latter is the situation of primary interest for the present study. In most of the previous modelling works dealing with the situations where vapour is present inside the wick, e.g. [13-18], only two steady-state 


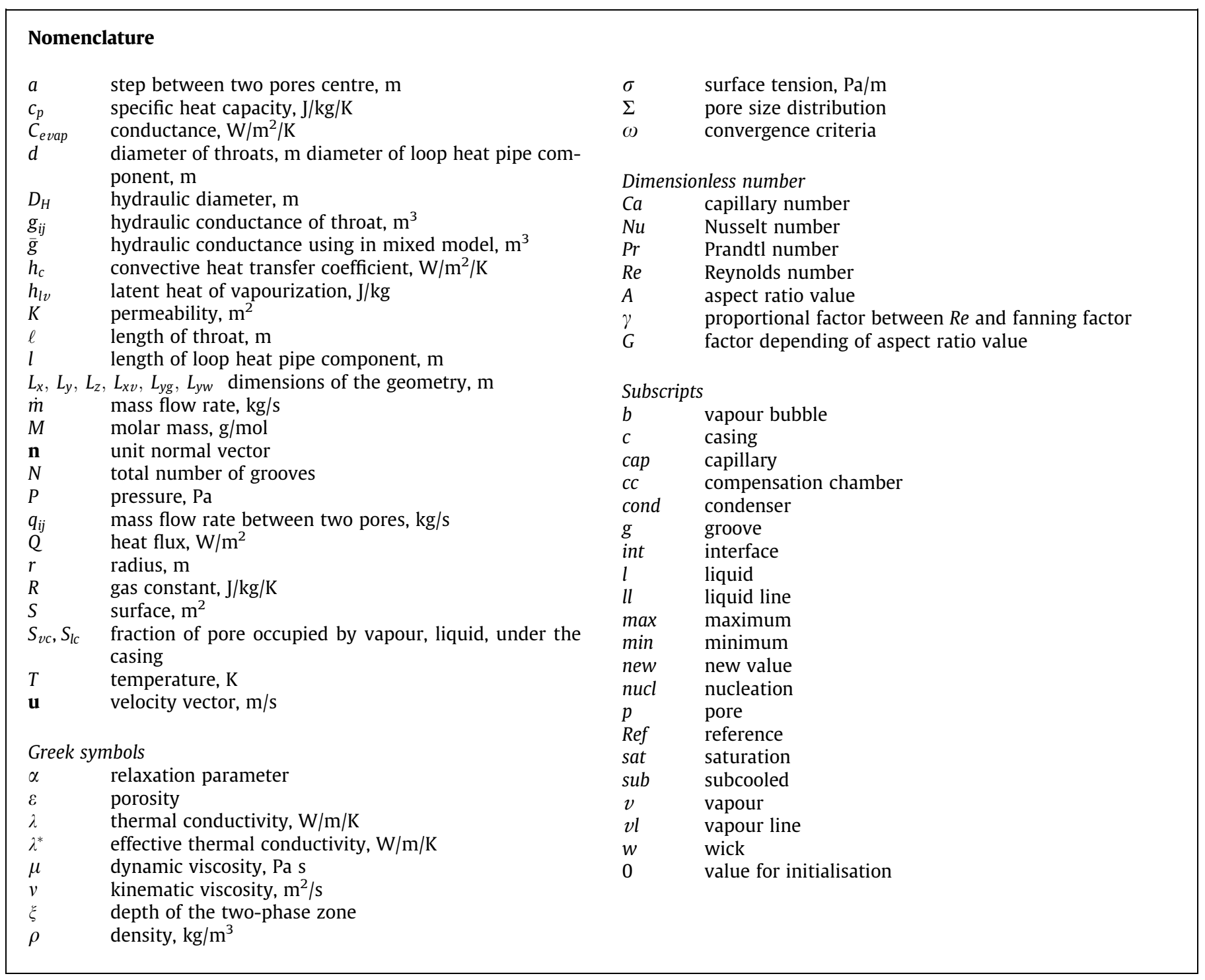

regimes were distinguished. For low to moderate heat loads, the wick was assumed to be fully saturated and vapourization took place at the wick/groove interface. Above a certain heat load, bubble nucleation occurred within the wick, leading to the formation of an internal vapourization front as sketched in Fig. 3. All these works were more or less implicitly based on the so-called "vapour pocket" assumption when vapour was present in the wick. As illustrated in Fig. 3, the "vapour pocket" assumption essentially amounts to assuming only two types of region within the wick: a fully dry region in contact with the casing occupied by the vapour and a fully liquid region elsewhere with a sharp interface between the two regions. This is in contrast with the work presented in [19], where it was considered that a two-phase zone, i.e. a zone where liquid and vapour coexists in the same region, formed within the wick. This was for moderate heat fluxes. For higher heat fluxes, a fully dry region could form but a two-phase zone was still present in the wick. However, the porous structure considered by [19] was held vertically, $40 \mathrm{~mm}$ in height and formed by large beads (on the order of $1 \mathrm{~mm}$ in diameter) and therefore operated in the presence of significant gravity effects. Thus, under conditions presumably
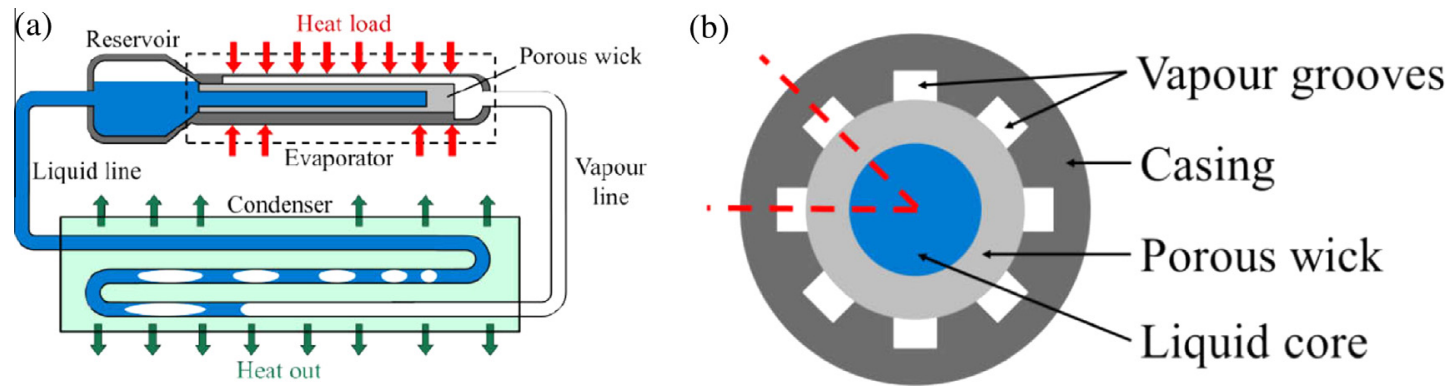

Fig. 1. (a) Schematic of a LHP (b) Schematic of the cross section of a cylindrical evaporator. The dashed lines define the evaporator unit cell. 

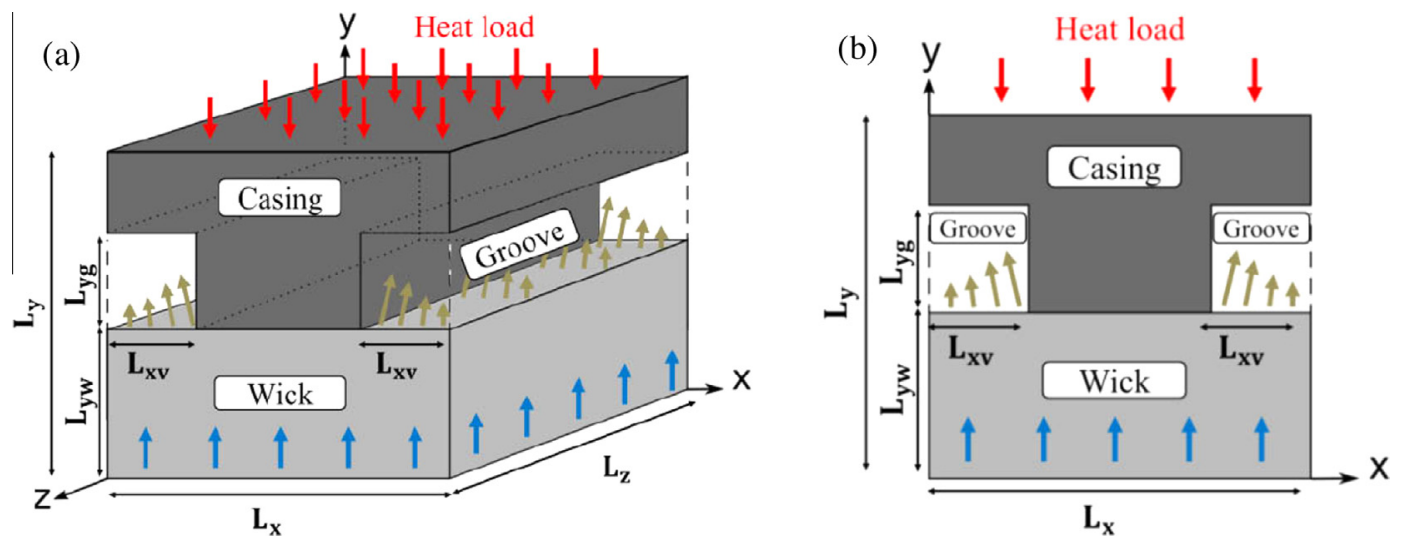

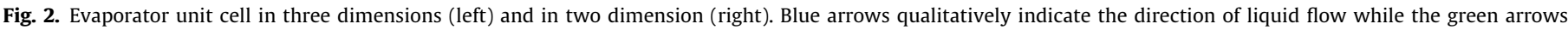
correspond to a vapour flow. (For interpretation of the references to colour in this figure legend, the reader is referred to the web version of this article.)

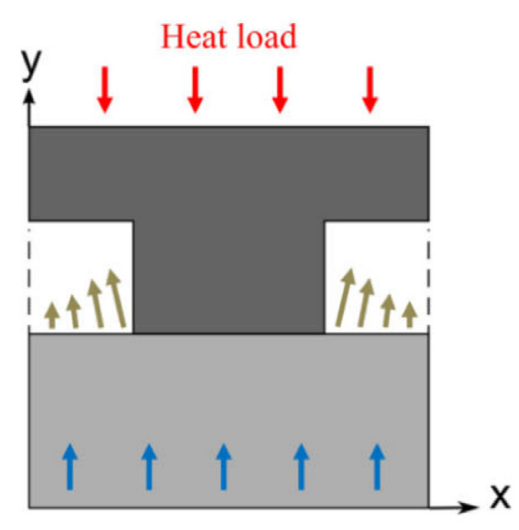

(a) Liquid saturated wick

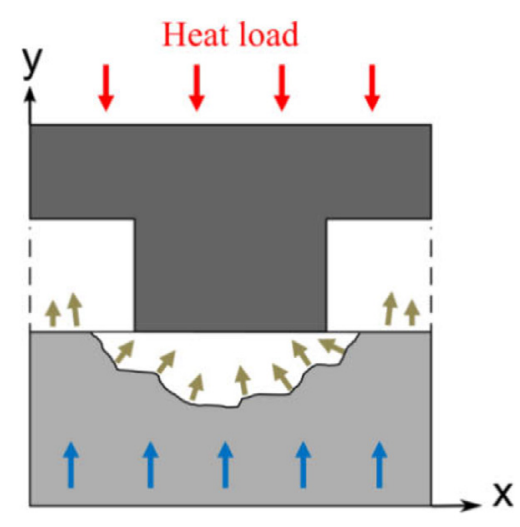

(b) Vapour-liquid wick

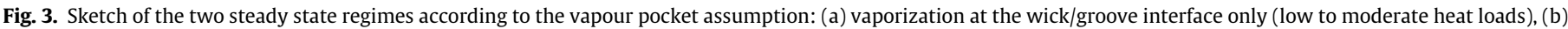

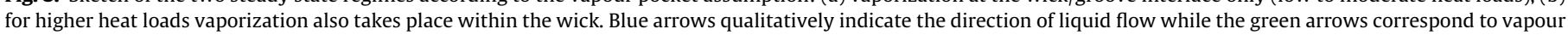
flow. (For interpretation of the references to colour in this figure legend, the reader is referred to the web version of this article.)

quite different from the ones prevailing in capillary evaporators. The formation of a two-phase zone within the wick was also predicted by [20] using a Darcy's scale two-phase flow model. However, the heat flux was directly applied at the wick surface without taking into account the heat transfer in the casing and therefore the fined shape of casing.

It is also worth mentioning that the results reported in [14] and some subsequent works, e.g. [18,21], leading to the formation of a vapour pocket (and not a two-phase zone) were obtained from simulations with two-dimensional pore network models (PNM). Actually, the gas-liquid phase distribution in a 3D microstructure can be expected to be fundamentally different from the 2D microstructures considered in [14]. This is because of the fundamentally different percolation properties of the phases between 2D pore networks and 3D pore networks, e.g. [22] and references therein. The essential point is that the gas and liquid phases can both form percolating clusters in 3D microstructures whereas this cannot happen in 2D random microstructures. In other words, the formation of the vapour pocket depicted in Fig. $3 \mathrm{~b}$ is fully consistent with the percolation properties of the 2D pore networks used in [14]. The phase distribution can be expected to be different in a 3D microstructure, which is of course the situation of interest in real evaporator wicks. The objective of the present work is therefore to study the much more representative and potentially fundamentally different 3D case.
As in [14], the work is based on pore network (PN) simulations. The crucial difference is the consideration of a 3D pore network in the present paper. Also, several new features have been introduced: the conductive heat transfer in the external casing is computed, the convection heat transfer is taken into account within the wick in addition to heat conduction and phase change, the variations of density in the vapour phase with pressure and temperature are taken into account assuming an ideal gas behaviour, the heat loss due to the vapour convective flow in the vapour grooves is taken into account.

Figus et al. [14] developed a pore network model in which the transfers between two nodes classically involve heterogeneous hydraulic or thermal local conductivities depending on the local geometrical dimensions of the constrictions between two pores, see for example [23] for more details on the pore network approach. This implies to know the local conductivity distributions or how the local conductivities are related to the constriction geometry. For a given porous medium these data are generally more difficult to obtain than effective transport properties, such as the porous medium permeability or its effective thermal conductivity. The latter are often determined by the porous material manufacturers or can be found in the literature. For this reason, a mixed formulation has been preferred for the present work. Transfers are described using equations involving macroscopic parameters such as the permeability or the effective thermal conductivity 
whereas we rely on the pore-network representation for accounting for the capillary effects. It could be argued that the mixed formulation is not consistent in terms of scale of description since capillary effects are treated at pore scale whereas transport seems to be considered at the R.E.V. (Representative Elementary Volume) scale, e.g. [24]. Actually, one should realise that the finite volume discretized forms of the volume averaged equations are very close to the resistance network equations considered in the classical pore network approach. This is made clear later in the paper. In other terms, the mixed pore network model used in the present work must be seen as a mesoscale approach, i.e. intermediate between direct simulations at pore scale and Darcy's scale model, where coarse-grained approximations are used to model the flows and the heat transfer.

The paper is organised as follows. The numerical model is presented in Sections 2 and 3. In Section 4, results from a visualisation experiment in a quasi-2D porous microstructure are presented. Section 5 presents a comparison between 2D numerical simulations and the experimental results. 3D simulations are presented in Section 6.

Thus, we will qualitatively validate the pore network model from 2D simulations and not 3D simulations. The rationale is as follows. Experiments with quasi-2D structures make the visualisations of the vapour and liquid phase distributions within a porous microstructure considerably easier. Then, if the pore network model is judged as validated in 2D, it can be used with much more confidence to perform 3D simulations.

\section{Evaporator unit cell numerical model}

As sketched in Fig. 4, the porous microstructure is represented by a structured pore network, a square network in 2D and a cubic pore network in 3D. In this type of model, the pore space is represented by a network of sites (also called pores) corresponding to the nodes of pore network. The pores are interconnected by channels (also called bonds or throats) representing the pore space constrictions. The throats are cylindrical tubes, whose diameter $d$ is randomly distributed according a given probability distribution function (p.d.f) between two bounds $d_{\min }$ and $d_{\max }$. A uniform p.d.f. is used in the present work unless otherwise mentioned. The distance between two nodes, denoted by $a$, is the lattice spacing. It also corresponds to the discretization step used to solve the equations governing the flow and heat transfer in the computational domain. Ideally, the lattice spacing should correspond to the mean distance between two pores in the material forming the wick. However, this would lead to a number of computational nodes making the simulations much too long, at least in 3D and with the present version of the code which is not parallelized. Thus, we use in practice a lattice spacing greater than the mean

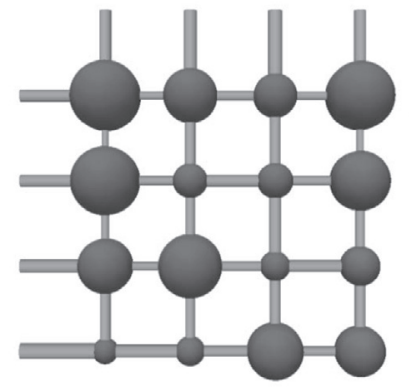

(a) 2D square network

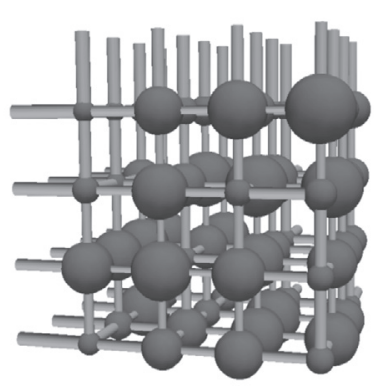

(b) 3D cubic network
Fig. 4. Pore network representation of porous microstructure. distance between two pores inside a real wick for the 3D simulations. This point is discussed further below in Section 6.1.

Computational nodes are also located in the casing using the same discretization step for computing the heat transfer by conduction in the casing. Under the assumptions of homogeneous and isotropic capillary structures, negligible gravitational and radiative effects, local thermal equilibrium between the porous structure and the working fluid, the problem to be solved within the framework of the mixed pore-network model can be expressed as follows (for the most complicated case, i.e. in the presence of vapour in the wick. The situation where the wick is fully saturated by liquid is a simpler sub-case). Note that we are only interested in steady state solutions.

\subsection{Flow in porous wick}

In the traditional pore network approach, the mass flow rate $q_{i j}$ between two adjacent nodes $i$ and $j$ is expressed as

$q_{i j}=\rho \frac{g_{i j}}{\mu}\left(P_{i}-P_{j}\right)$

where $\rho$ and $\mu$ are the density and dynamic viscosity of the fluid (vapour or liquid) occupying the throat joining the two pores; $P_{i}$ ( $P_{j}$ respectively) is the pressure in pore $i$ ( $j$ respectively). The hydraulic conductance $g_{i j}$ is generally expressed assuming a Poiseuille flow in the throat,

$g_{i j}=\frac{\pi}{128} \frac{d_{i j}^{4}}{\ell}$

where $\ell$ is the throat length and $d_{i j}$ the size (diameter) of the considered throat.

The approximation made with the mixed model approach is to use the same hydraulic conductance $\bar{g}$ for all the throats. This amounts to expressing Eq. (1) as:

$q_{i j}=\rho \frac{\bar{g}}{\mu}\left(P_{i}-P_{j}\right)$

Hence, the approximation can be regarded as a mean field approximation of the flow in the considered disordered porous structure.

Consider now the classical Darcy model of the flow in a porous medium,

$\nabla \cdot(\rho \mathbf{u})=0$
$\mathbf{u}=-\frac{K}{\mu} \nabla P$

where $K$ is the porous medium permeability.

Considering a square (2D) or a cubic (3D) regular mesh of spatial step $a$, the classical finite volume discretization of Eqs. (4) and (5), e.g. [25], leads to express the flow between two adjacent nodes $i$ and $j$ as

$q_{i j}=\rho u a^{2}=\rho a^{2} \frac{K}{\mu} \frac{P_{i}-P_{j}}{a}$

Comparing Eqs. (6) and (3) then leads to express the mean field hydraulic conductance as

$\bar{g}=a K$

Then the flow in the region of the wick occupied by the liquid (the vapour respectively) is computed from the mass balance expressed as each liquid (vapour respectively) nodes,

$\sum_{j} q_{i j}=0$

where $j$ corresponds to the four (six respectively) pores adjacent to pore $i$ in a 2D network (in a 3D network respectively). 
Combined with the boundary conditions given below, Eq. (8) leads to a linear system of equations whose solution gives the pressure in each pore.

The pore network approach using the mean field conductance $\bar{g}$ or the approach based on the finite-volume discretized form of the Darcy's model are therefore fully equivalent. In what follows, we proceed directly with the Darcy's formulation. The liquid phase is considered as incompressible whereas an ideal gas behaviour is assumed for the vapour.

$\rho_{v}=\frac{P M}{R T}$

\subsection{Heat conduction in casing}

$\nabla \cdot\left(\lambda_{c} \nabla T\right)=0$

where $T$ is the temperature and $\lambda_{c}$ is the casing thermal conductivity. Similarly as the flow problem, this equation is discretized using a standard finite volume approximation on a regular square or cubic mesh.

\subsection{Heat transfer in porous wick}

Transfer is considered to occur by convection and conduction in the wick

$\left(\rho_{i} c_{p, i}\right) \mathbf{u}_{i} \cdot \nabla T=\nabla \cdot\left(\lambda_{i}^{*} \nabla T\right)$

with $i=l$ or $v$ depending on the fluid occupying the considered node. In Eq. (11), $\left(\rho c_{p}\right)$ is the heat capacity of the porous medium occupied either by liquid or vapour, $\lambda_{i}^{*}$ is the effective thermal conductivity of the medium which also depends on the fluid occupying the considered node. Again Eq. (11) is discretized using a standard finite volume approach.

\subsection{Boundary conditions}

Spatially periodic boundary conditions are imposed on the lateral sides of computational domain. The boundary condition at the casing external surface is expressed as:

$\left(\lambda_{c} \nabla T\right) \cdot \mathbf{n}=Q$

where $\mathbf{n}$ is the interface normal unit vector and $Q$ the heat flux applied on the casing.

No flow condition and energy conservation condition are imposed at the wick-casing interface,

$\nabla P \cdot \mathbf{n}=0$

$\left(-\lambda_{i}^{*} \nabla T\right) \cdot \mathbf{n}=\left(-\lambda_{c} \nabla T\right) \cdot \mathbf{n}, \quad i=l$ or $v$

Convection heat transfer is assumed at the casing/groove interface:

$\left(\lambda_{c} \nabla T\right) \cdot \mathbf{n}=h_{c}\left(T-T_{g}\right)$

where $T_{g}$ is the temperature in the groove, the convective heat transfer coefficient $h_{c}$ is computed using the correlation presented in [26], see Appendix A for details. The approximation is made that the temperature in the groove is close to the saturation temperature at the pressure $P_{g}$ in the groove,

$T_{g} \approx T_{\text {sat }}\left(P_{g}\right)$

where $P_{g}$ is computed using the loop model presented in [9]. It can be expressed as:

$P_{g}=P_{c c}+\Delta P_{\text {loop }}$

where $P_{c c}$ and $\Delta P_{\text {loop }}$ are the pressure in compensation chamber and pressure drop in the other components of the loop. The details on how $\Delta P_{\text {loop }}$ is computed are given in Appendix B.
The pressure and the temperature are specified at the entrance of the wick (wick/liquid-passage core interface). They correspond to the pressure and the temperature in the compensation chamber:

$T=T_{\text {sat }}-\Delta T_{\text {sub }}, \quad P=P_{\text {sat }}\left(T_{\text {sat }}\right)=P_{c c}$

where $\Delta T_{\text {sub }}$ is the subcooling. The liquid leaving the compensation chamber is generally slightly subcooled. The degree of subcooling of working fluid is used to compensate the heat leak (the fraction of the applied heat flux which is not used for the vapourization of the liquid). The subcooling originates from what happens in the condenser. There are generally three zones within the condenser: the vapour zone, the two-phase zone and the liquid zone. In the liquid zone, the liquid phase of the working fluid is completely condensed, resulting in the removing of the last vapour bubbles. At the end of the condenser, the liquid temperature is thus below the saturation temperature. The liquid stays subcooled through the liquid line. The mixing between the subcooled liquid arriving from the liquid line into the compensation chamber and the liquid present within the compensation chamber results in a slightly subcooled liquid at the entrance of the evaporator. Another interest of the subcooling is to prevent the formation of bubbles within the compensation chamber. If vapour occurs at the inlet of the evaporator, vapour bubbles could accumulate and block the path for the liquid at the inlet of the porous wick resulting in a deprime of the evaporator. The subcooling at the inlet of the wick is generally small and is neglected in the simulations presented later in the paper.

Of special importance are the boundary or interfacial conditions where the liquid-vapour phase change can take place; namely the groove-wick interface as well as the liquid-vapour interface inside the wick when the wick is partially invaded by the vapour.

The boundary condition at the porous wick-groove interface is expressed using Eq. (19) when liquid is present at the considered point of this interface. Eq. (20) is used when the computational point corresponds to a region occupied by vapour at the interface.

$\left(-\lambda_{l}^{*} \nabla T\right) \cdot \mathbf{n}=\rho_{l} h_{l v} \mathbf{u}_{l} \cdot \mathbf{n}$

$\left(\lambda_{l}^{*} \nabla T\right) \cdot \mathbf{n}=h_{c}\left(T-T_{g}\right)$

In Eq. (19), $h_{l v}$ is the latent heat of vapourization.

The flow continuity and energy conservation conditions at the vapour-liquid interface within the wick are expressed as:

$\rho_{v} \mathbf{u}_{v} \cdot \mathbf{n}=\rho_{l} \mathbf{u}_{l} \cdot \mathbf{n}$

$\left(-\lambda_{l}^{*} \nabla T\right) \cdot \mathbf{n}-\left(-\lambda_{v}^{*} \nabla T\right) \cdot \mathbf{n}=\rho_{l} h_{l v} \mathbf{u}_{l} \cdot \mathbf{n}$

$T_{l}=T_{v}=T_{\text {sat }}\left(P_{v}\right)$

The Clausius-Clapeyron relationship:

$P_{\text {sat }}\left(T_{\text {sat }}\right)=P_{\text {ref }} \exp \left(-\frac{h_{l v} M}{R}\left[\frac{1}{T_{\text {sat }}}-\frac{1}{T_{\text {ref }}}\right]\right)$

is used to link the temperature and the vapour pressure along the liquid-vapour interface.

Capillarity is a crucial phenomenon in the evaporator wick. As sketched in Fig. 5, the liquid-vapour interface is actually discrete and formed by a series of menisci located at the entrance of throats.

The maximum pressure difference between the liquid and the vapour across such a meniscus is given by Laplace's law,

$\Delta P_{\max }=P_{\text {cap }, \max }=\frac{4 \sigma}{d}$

where $\sigma$ is the surface tension and $d$ the size of the considered throat. Note that the liquid is assumed to be perfectly wetting. 


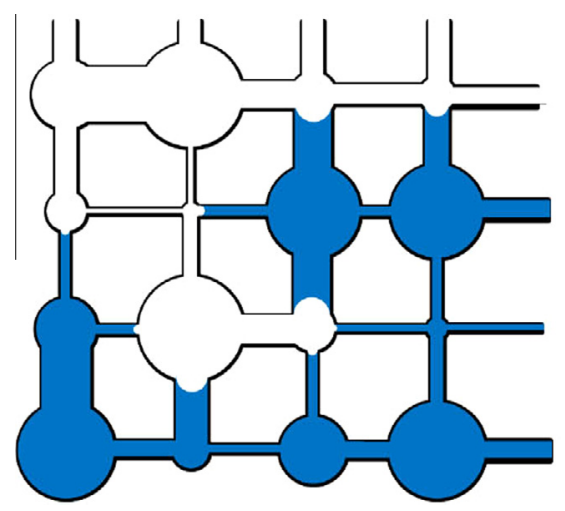

Fig. 5. Sketch of discrete liquid-vapour interface in pore network. Liquid phase in blue. Vapour phase in white. (For interpretation of the references to colour in this figure legend, the reader is referred to the web version of this article.)

\section{Method of solution}

The above equations are solved using a home-made code written in Fortran90. As mentioned before, the discretization of governing equations is performed using standard finite volume procedure. An iteration procedure is necessary to obtain the steady state solution. This is obvious when vapour enters the wick since the position of the liquid-vapour interface is not known a priori. However, an iterative procedure is also needed when the wick remains fully saturated by the liquid. This is because the solution depends on the pressure drop in the other components of the loop through Eq. (17). The iterative method for the case with a saturated wick is summarised in Fig. 6.

Let's now consider the procedure for the situations where vapour partially invades the wick. A priori, one can distinguish two possible scenarios depending on the pores first invade by vapour. The first scenario would correspond to the situation where the pressure jump across some menisci at the wick/groove interface exceed the maximum capillary pressure given by Laplace's law, Eq. (25). Such a meniscus could not stay at the wick/groove interface and should recede into the wick. The second scenario is the possible nucleation of vapour in pores. According to nucleation theory this is possible when the temperature becomes sufficiently high in some pores for nucleation to occur. In this case, pores can be invaded by vapour not necessarily at the wick-groove interface but inside the wick. The temperature $T_{n u c l}$ marking the onset of nucleation can be expressed as,

$T_{\text {nucl }}=T_{\text {sat }}\left(P_{v}\right)+\Delta T_{\text {nucl }}$

where $\Delta T_{\text {nucl }}$ is the nucleation superheat, which depends on several factors such as the operating conditions, the nature of the fluid, the porous material, the pore wall topography, etc. To make use of Eq. (26), one also need to specify $P_{v}$. A simple procedure is to take $P_{v}=P_{g}$. A potentially more interesting approach is to take for $P_{v}$ the pressure corresponding to the formation of a bubble of radius $r_{b}$ in a pore. Hence,

$P_{v}=P_{l}+\frac{2 \sigma}{r_{b}}$

where $P_{l}$ is the liquid pressure in the pore prior nucleation, i.e. the pressure computed assuming a fully saturated zone. A problem is to estimate the bubble radius $r_{b}$ since $r_{b}$ is supposed to correspond to the minimum size of embryo leading to the bubble formation. In the absence of more information, a simple rule is to take $r_{b} \approx r_{p}$ where $r_{p}$ is the pore radius. The advantage of this approach is to make the nucleation criterion depending on the local liquid pressure. This will be discussed further from the numerical results presented in
Section 5. A difficulty remains: the evaluation of $\Delta T_{\text {nucl }}$. This is still an open problem. As in previous works, i.e. [14,18], and in accordance with the discussion in [15], a few Kelvin superheat is assumed. We took $\Delta T_{\text {nucl }}=4 \mathrm{~K}$ for all the simulations reported in the present paper. With a superheat of a few degrees, the occurrence of the vapour within the wick is then due to the nucleation of vapour bubbles at the wick-casing interface and not to the receding of menisci located at the wick-groove interface according to our simulations. The receding of menisci located at the wick-groove interface do happen in our simulations but only after a liquidvapour two-phase zone has formed within the wick.

The procedure is then as follows after the nucleation step. The model cannot handle vapour pressurization steps, which can occur as long as the vapour has no reached the grooves when nucleation take place inside the wick. Actually, simulations show that nucleation takes place at the casing-wick interface which is the place of highest temperatures in the wick. Starting from the node corresponding to $\max \left(T-T_{\text {nucl }}\right)$ with of course $T-T_{\text {nucl }}>0$, a vapour cluster percolating to one of the two grooves is formed by using the standard invasion algorithm, [27], in the 2D layer of pores in contact with the casing. When the simulation is performed using a two-dimensional pore network, this amounts to imposing the first row of pores under the casing as occupied by vapour. The distribution so-obtained is the initial guess for the computation of the liquid-vapour interface within the wick. Solving Eqs. (1)-(27) gives the temperature and pressure fields in both the liquid phase and the vapour phase within the wick. Then we compute the pressure jump $\Delta P=P_{v}-P_{l}$ across each meniscus present within the wick or at the wick/groove interface. Each meniscus such that its pressure jump is greater that its capillary pressure threshold, i.e. such that $\Delta P>\Delta P_{\max }$, is moved into the adjacent pore. This gives a new liquid-vapour phase distribution within the wick. Equations (1)(27) are then solved again until convergence for this new phase distribution and stability of menisci is tested as explained before, leading possibly to new pore invasions. This procedure is then repeated until the criterion of meniscus stability, i.e. $\Delta P \leqslant \Delta P_{\max }$, is fulfilled for each meniscus present in the wick.

Actually, the procedure can be performed using various rules as regards the moving menisci. For example, one can move at each step only one meniscus, the less stable one corresponding to $\max \left(\Delta P-\Delta P_{\max }\right)$. On the contrary, we can move all the menisci such that $\Delta P-\Delta P_{\max }>0$ or some fraction of these menisci. Tests indicate that the obtained steady-state solution is only slightly depending on this choice. Since the solution is not very sensitive to this aspect of the procedure, the procedure leading to the shortest computational times is adopted, namely the one where all menisci detected as unstable are moved at each step.

The iterative method leading to the solution for an unsaturated wick is summarised in Fig. 7.

\section{Visualisation experiment}

\subsection{Experimental set-up}

The quasi-two-dimensional micromodel developed in order to perform visualisation of the invasion of the wick by vapour is shown in Fig. 8. It mimics the unit cell of a capillary evaporator. The porous medium is formed by a monolayer of steel beads of $1 \mathrm{~mm}$ in diameter glued on a $2 \mathrm{~mm}$ thick brass support plate. The beads are randomly distributed so as to create a pore size distribution similarly as in real porous material. The metallic casing is in brass and is linked to an electric heating element to impose a heat flux as sketched in Fig. 8.

These elements are sandwiched between two Plexiglas plates. As can be seen from Fig. 8, the model unit cell is linked to a reservoir 


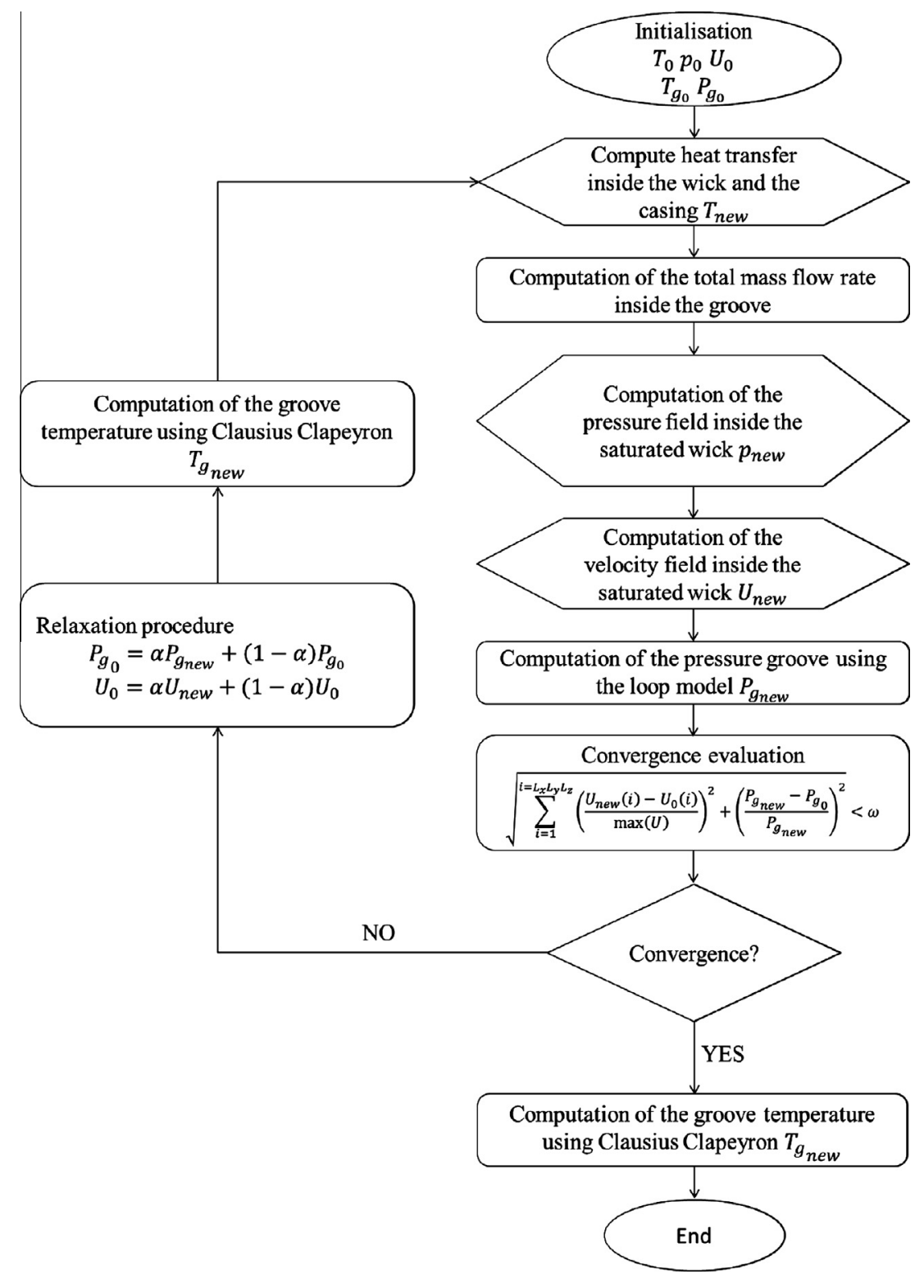

Fig. 6. Computational chart for the case with a liquid saturated wick.

feeding the wick in liquid. Two tubes, referred to as the vapour lines, are linked to the two vapour chambers representing the grooves to collect the vapour produces inside the micromodel. The vapour lines are horizontally positioned on the side of the grooves. The vapour condenses along the vapour lines and is collected in a reservoir. The latter is placed on a weighing scale to determine the mass of fluid leaving the system.

The working fluid is the ${ }^{3 \mathrm{M}}$ Fluorinert ${ }^{\mathrm{TM}} \mathrm{FC} 3284$. Its saturation temperature is $50{ }^{\circ} \mathrm{C}$ at the atmospheric pressure. As shown in Fig. 9, this system is placed inside a temperature controlled enclosure. The experiments were carried out with a temperature fixed at $42{ }^{\circ} \mathrm{C}$ in the enclosure. A high definition camera $(2000 \times 2000$ pixels) is used to visualise and record the evolution of the phase distribution within the wick. The permeability, porosity and capillary pressure curve (from which a pore size distribution can be determined) of the system have been determined experimentally. The details are presented in [28]. The various properties characterising this model system are summarised in Table 1.

Dimensions of the model evaporator unit cell are indicated in Fig. 8. These dimensions are about one order of magnitude greater than in a real LHP evaporator unit. Also the throat sizes, on the order of $100 \mu \mathrm{m}$, are between one and two orders of magnitude greater that in the wicks of LHP evaporators. However, the important point is not to have the same dimensional properties as in LHP evaporators but the same forces controlling the phase distributions, namely the capillary and viscous forces (the model unit cell being hold horizontally gravity effects are negligible in the experiments). Also the objective is not a truly quantitative comparison between experiments and simulations since there are some heat losses through the upper plate of model unit cell, which are unavoidable and difficult to control with such a quasi-two dimensional system. Despite its imperfection, we believe that this experiment is nevertheless instructive as we shall also see from the comparison with simulations.

The experimental procedure is as follows. The system is first fully saturated by degasified liquid Fluorinert. Then the heat flux is applied and the images showing the evolution of the fluid phases resulting from the application of heat flux are recorded. Experiment is considered as finished when the vapour breakthrough is reached, i.e. when vapour percolates through the wick. 


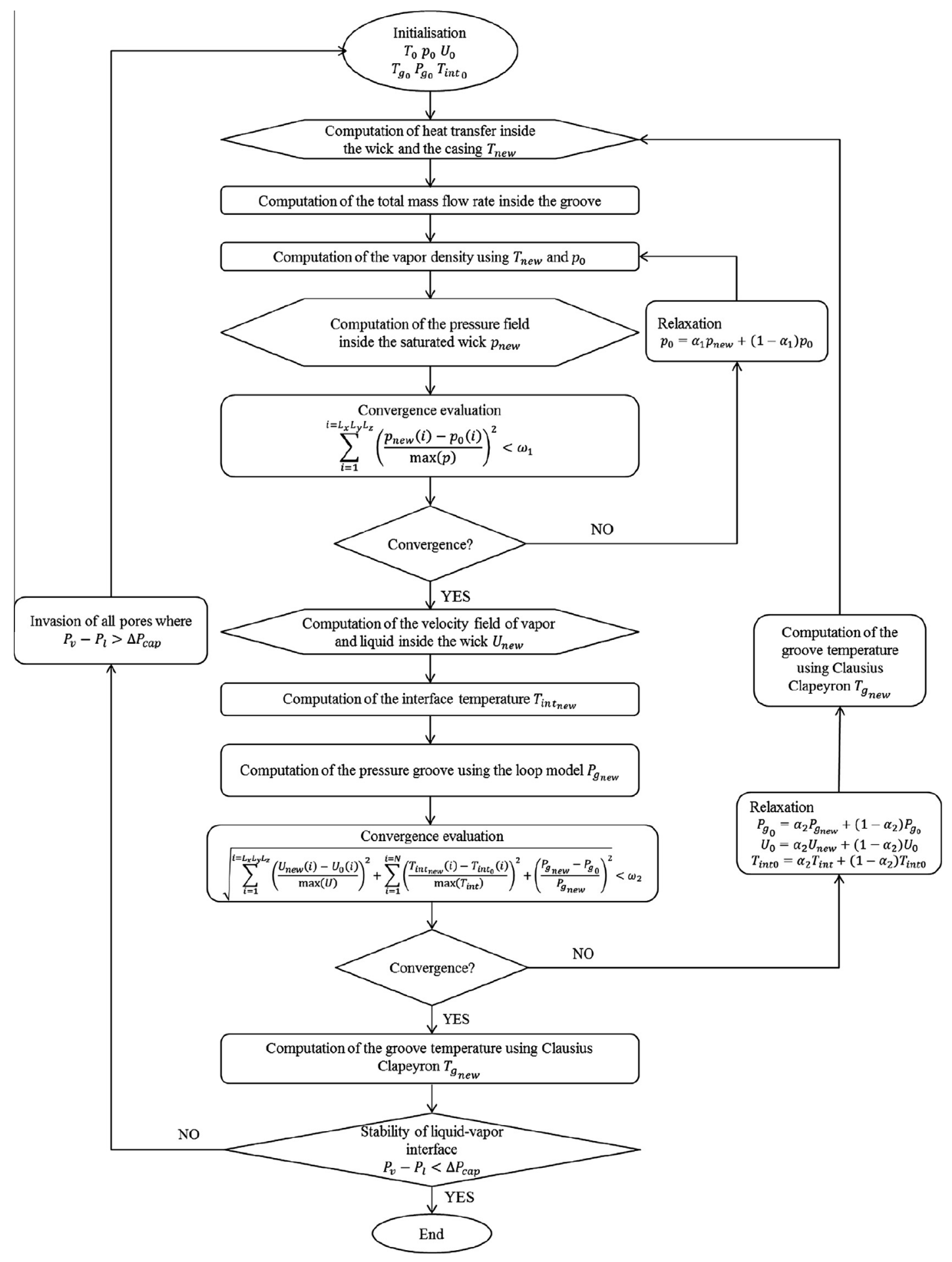

Fig. 7. Computational chart for the case with an unsaturated wick.

\subsection{Experimental results and discussions}

Two micromodels (referred to as micromodel A and micromodel B) were actually used. They differ only by the random distribution of the beads. Tests were performed with micromodel $\mathrm{A}$ and micromodel B with an applied flux of $2 \mathrm{~W}$ (test \#1). Figs. 10 and 11 show the growth of the vapour region in the wick with micromodel $A$ and micromodel $B$ respectively.

For micromodel A, invasion of the wick by the vapour starts relatively close to the casing/groove/wick contact line (Fig. 10a) and grows until reaching the other groove located on the other side of casing (Fig. 10b). This is in contrast with the assumption considered in where nucleation was supposed to occur in the middle of the wick/casing interface and not at the contact line with the grooves. Then vapour continues to invade the porous medium (Fig. 10c and d) keeping an approximate symmetric shape relative to the fin. At some point, the symmetry is broken (Fig. 10e) and the vapour continues to move toward the wick inlet. As shown in Fig. 10e, viscous fingers begin to appear and develop until vapour breakthrough (Fig. 10f). As shown in Fig. 11, the invasion of the 


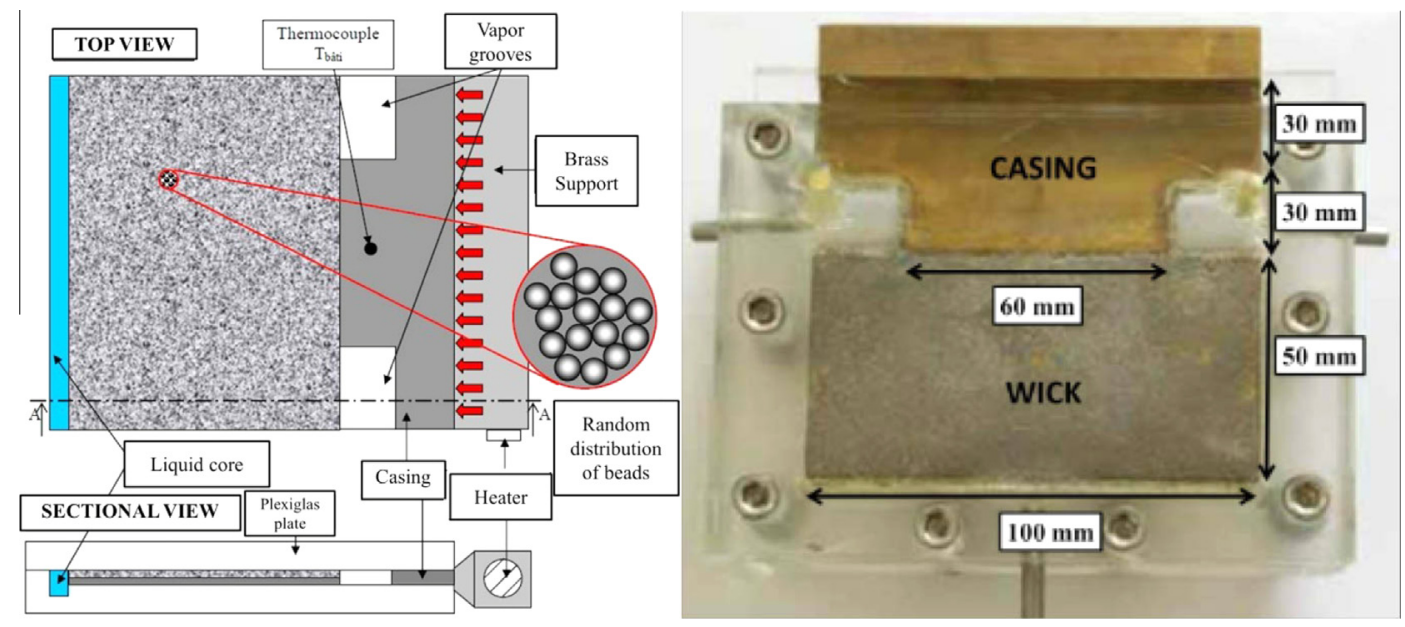

Fig. 8. Two-dimensional model evaporator unit cell.

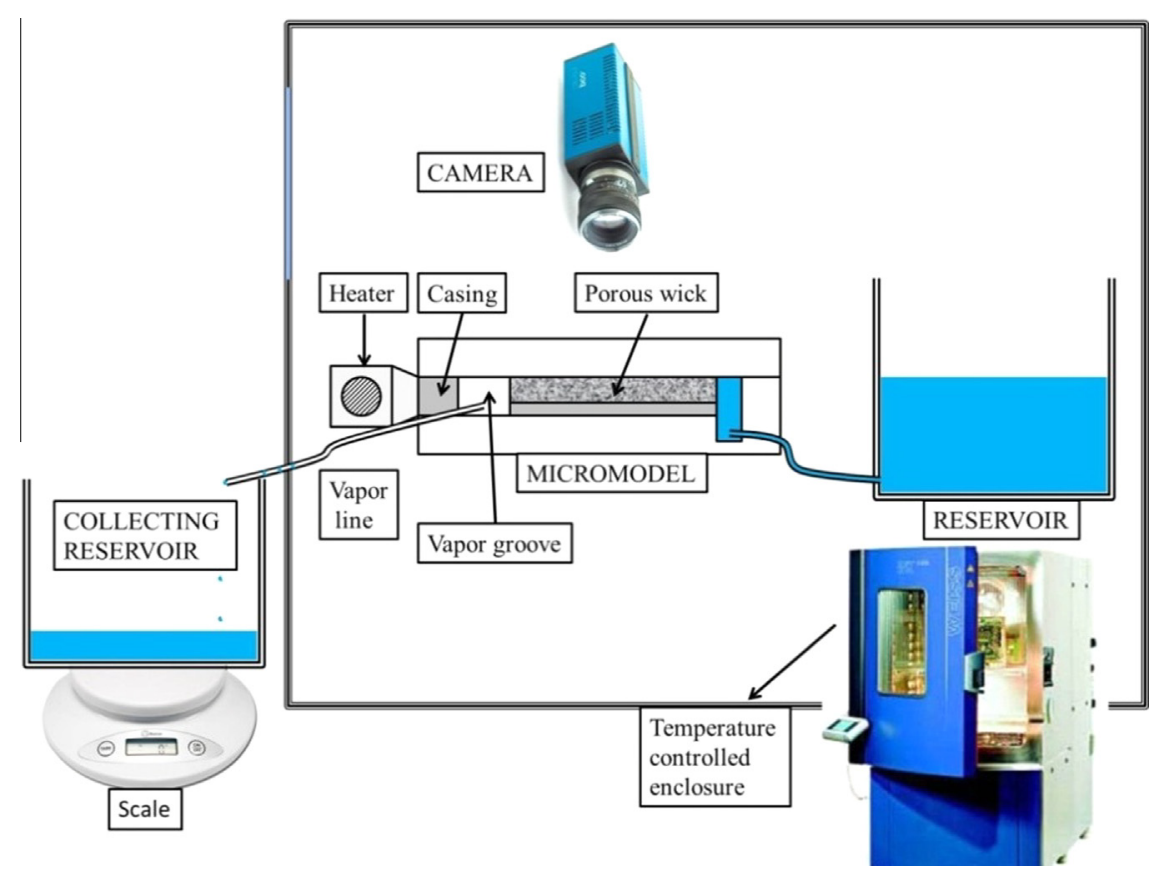

Fig. 9. Experimental setup.

Table 1

Fluid and model evaporator unit cell main properties.

\begin{tabular}{lll}
$\begin{array}{l}\text { Wick Properties } \\
\text { Porosity }\end{array}$ & $\varepsilon=0.52$ \\
$\begin{array}{l}\text { Conductivity of beats } \\
\text { Permeability }\end{array}$ & $\lambda_{\text {beats }}=46 \mathrm{~W} / \mathrm{m} / \mathrm{K}$ & \\
Casing properties & $K=968.10^{-11} \mathrm{~m}^{2}$ & \\
Conductivity of brass & & \\
Fluid properties & $\lambda_{c}=110 \mathrm{~W} / \mathrm{m} / \mathrm{K}$ & \\
& & \\
Conductivity & Liquid & Vapor \\
Dynamic viscosity & $\lambda_{l}=0.057 \mathrm{~W} / \mathrm{m} / \mathrm{K}$ & $\lambda_{v}=0.0095 \mathrm{~W} / \mathrm{m} / \mathrm{K}$ \\
Specific heat capacity & $\mu_{l}=7.10^{-4} \mathrm{~Pa} . \mathrm{s}$ & $\mu_{v}=2.10^{-5} \mathrm{~Pa} . \mathrm{s}$ \\
Latent heat of vaporization & $c_{p, l}=1045 \mathrm{~J} / \mathrm{kg} / \mathrm{K}$ & $c_{p, v}=600 \mathrm{~J} / \mathrm{kg} / \mathrm{K}$ \\
Liquid density & $h_{l v}=100,300 \mathrm{~J} / \mathrm{kg}$ & \\
Interfacial tension & $\rho_{l}=1670 \mathrm{~kg} / \mathrm{m}^{3}$ & \\
& $\sigma=0.009 \mathrm{~N} / \mathrm{m}$ & \\
\hline
\end{tabular}

wick by vapour is globally similar for micromodel B but there are also interesting differences. As shown in Fig. 11b, vapour invasion takes place from a nucleation region located at the wick/casing interface inside the wick far from the grooves. Thus not close to the casing-wick-groove triple line in contrast with micromodel A. Then the growth occurs toward the middle of the wick/casing interface and not toward the adjacent groove (Fig. 11b) with also some invasion toward the wick inlet. Then, the invasion continues approximately symmetrically until the vapour region connects to the grooves on each side (Fig. 11c and d). The vapour region takes a triangular shape (Fig. 11e) and grows more symmetrically (Fig. 11f) than in micromodel A. Some viscous fingers can be observed a little before vapour breakthrough but their spatial extension is less than with micromodel A. About $2 / 3$ of the porous medium is invaded by vapour when the vapour breakthrough occurs (Fig. 11f). 

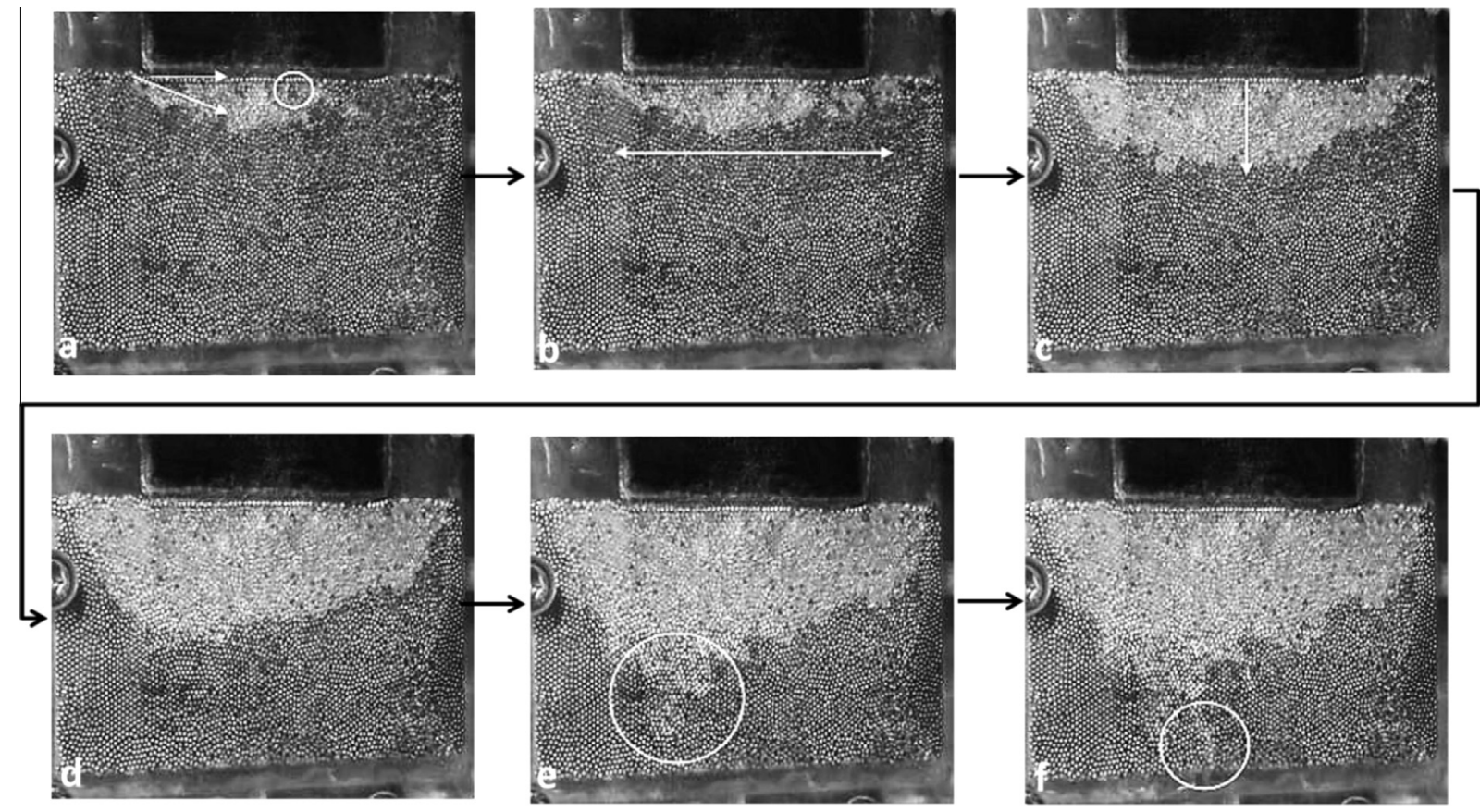

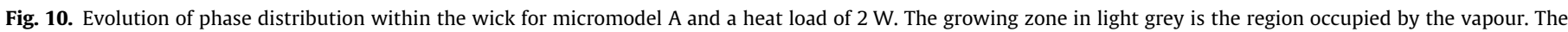
arrows schematically indicate the direction of vapour zone growth. A capillary-viscous vapour finger is visible in the circles, at breakthrough in Fig. 10f.
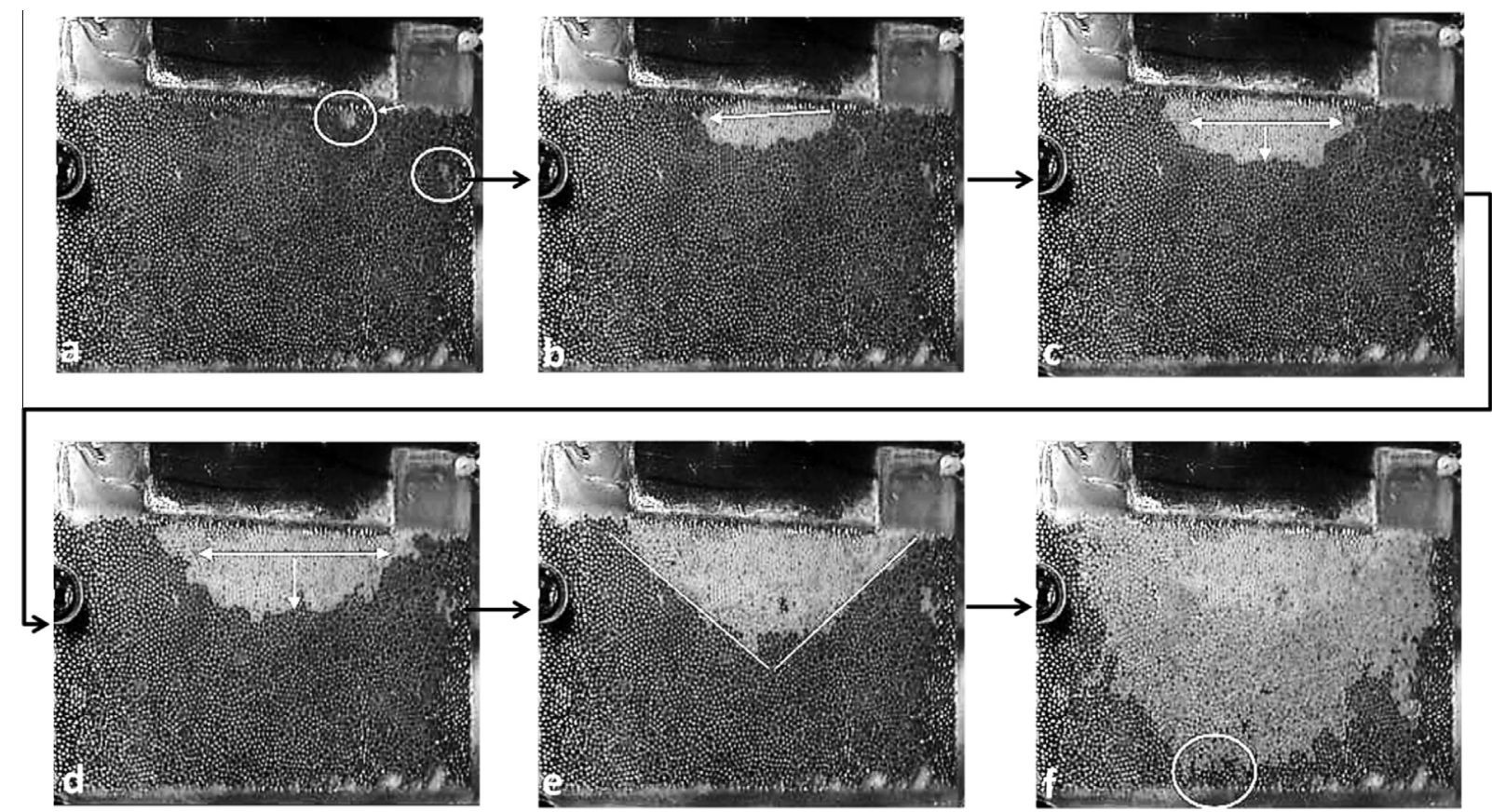

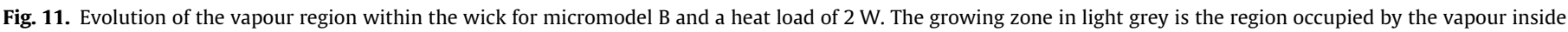

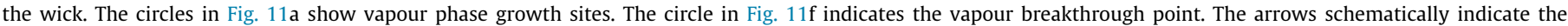
direction of vapour zone growth.

It can be noticed that we did not observe steady state regimes with a stabilized vapour region inside the wick with this experimental set-up, at least for the conditions we have tested. Nevertheless, we can summarise the main findings as follows. First the experiments provide images of phase distributions within the wick. Even if a truly steady state regime is not obtained, it will be interesting to compare these images with simulations. Second, the experiments indicate that vapour invasion can take place from about everywhere at the wick-casing interface, thus not necessarily from the hottest region in the wick, which is the region in the middle of this interface at equal distance from the two grooves. Third, the number of nucleation spots is small. Basically, only one nucleation spot is observed when the heat load is sufficiently low. Finally, we also note the occurrence of viscous fingers after 
a significant fraction of the wick has been invaded. Owing to the larger pore sizes compared to a real wick porous material, the capillary number, which characterises the competition between viscous and capillarity effects can be expected to be somewhat greater in the experiment compared to the situation prevailing in the wick of an operating LHP. The tentative conclusion is that the formation of viscous fingers is expected to be less likely in the wick of a LHP.

\section{Comparisons between two-dimensional simulations and experiment}

Experimental results for the micromodel $\mathrm{B}$ are chosen to be compared with simulations. The fluid and materials properties and the geometrical dimensions of the experiment (as listed in Table 1 and shown Fig. 8) are used as input data for the code. The simulations are performed in 2D with a lattice spacing of $1 \mathrm{~mm}$. Bonds diameters are randomly distributed in the range $0.55 \pm 0.44 \mathrm{~mm}$. according to a distribution law deduced from an image analysis technique, see [28] for the details. The flux lost by conduction through the brass support plate is approximately taken into account using as thermal conductivity of the solid phase $\lambda_{s}=0.5 \lambda_{\text {bead }}+0.5 \lambda_{\text {brass }}$. The effective thermal conductivity of the wick, which depends on the fluid occupying the considered region of the computational domain is then estimate as the arithmetic average between the classical estimates for arrangement of the phases in parallel and in series. The experimental heat load applied at the top of the casing is $2 \mathrm{~W}$, leading to an applied heat flux of $6700 \mathrm{~W} / \mathrm{m}^{2}$. As mentioned before, nucleation is supposed to occur if the temperature in the wick is greater than $T_{\text {sat }}+4 \mathrm{~K}$, where $T_{\text {sat }}$ is the saturation temperature at the atmospheric pressure $\left(T_{\text {sat }}=50^{\circ} \mathrm{C}\right)$. The inlet pressure is taken equal to the ambient pressure. A grid of $107 \times 54$ points is used for the wick corresponding to 5778 computation points in agreement with the number of beads inside micromodel B. A grid of $107 \times 32$ points ( $3424 \mathrm{com}-$ putation points) is used in the groove-casing region, leading to a total number of 9202 computational points.

Owing to the randomness of the bead distribution forming the porous medium in the experiment, the simulation is performed for 13 different realizations of pore network, i.e. 13 drawings of the bond size distribution using the same p.d.f. To characterise the invasion of the wick by the vapour, the length of the vapour region, defined as the ratio of the maximum depth of the vapour region to the total depth of the wick, the vapour saturation, which is the fraction of the pores occupied by vapour, and the occupancy ratio of vapour at wick/grooves interface, which is defined as the ratio of the number of pores occupied by vapour along this interface to the total number of pore along this interface, are computed. The simulation results are reported in Table 2 and compared to experimental data. As can be seen from Table 2, the average values obtained from the simulations are in reasonable agreement with the experimental data. Simulation \#5 is the one leading to the closest results with the experiment. Vapour breakthrough is observed in the experiment for the considered applied heat load and the experimental data reported in Table 2 correspond to the phase distribution observed when the vapour breakthrough occurs in the experiment. Numerical simulations show that the vapour breakthrough can also occur depending on the considered realization. This happens with realizations \#1, 4, 6 and 12. Furthermore, the depth of the vapour region is high for the other realizations. Although a stable interface is obtained for those realizations, the phase distribution is actually close to breakthrough. Examples of computed phase distribution are shown in Fig. 12. As can be seen, the obtained shape of the vapour region is qualitatively similar to the shapes observed in the experiments.
Another interesting point is to determine where the first vapour cluster forms within the wick. Fig. 13 shows the computed temperature distribution along the casing-wick interface for an applied flux of $0.5 \mathrm{~W} / \mathrm{cm}^{2}$. This flux is lower than the flux of $0.67 \mathrm{~W} / \mathrm{cm}^{2}$ applied in the experiment. It corresponds to a flux where the temperature computed under the casing is on the order of the assumed nucleation temperature. Imposing the heat flux of $0.67 \mathrm{~W} / \mathrm{cm}^{2}$ leads to temperatures under the casing much greater than the nucleation temperature, consistently with the fact that this flux leads to a major invasion of the model wick by the vapour. The temperature distribution shown in Fig. 13 is assumed to be representative of the temperature distribution when nucleation starts in the wick when the transient flux seen by the wick is still below the applied heat flux of $0.67 \mathrm{~W} / \mathrm{cm}^{2}$. Note that the parabolic shape depicted in Fig. 13 does not depend on the applied flux. Hence the hottest point is always found in the middle of casing when the wick is liquid saturated. Pores can become vapour when the temperature at wick/casing interface is higher than $T_{\text {nucl }}$. The results shown in Fig. 13 thus indicate that the most likely place for nucleation if right underneath the casing more or less in the middle of the casing-wick interface. However, it can be noted that the temperature variation along the casing/wick interface is quite low, less than $0.5 \mathrm{~K}$ except on the edges. Thus it can be considered that nucleation can actually take place about everywhere at the casing/wick interface, in agreement with the experiment.

We conclude that the comparisons presented in this section are sufficiently satisfactory to use with confidence the mixed pore network model for studying the heat and mass transfer with phase change inside the porous wick of a capillary evaporator.

\section{Three-dimensional simulations}

\subsection{Liquid-vapour distribution within the wick}

In this section, results of simulations performed considering the $3 \mathrm{D}$ evaporator unit cell are presented. The geometrical dimensions and porous medium properties are indicated in Table 3. They are representative of a LHP evaporator unit cell. The working fluid is ammonia. Its physical properties are also indicated in Table 3. No subcooling is considered, i.e. $\Delta T_{s u b}=0 \mathrm{~K}$.

Nucleation is supposed to occur if the temperature in the wick is greater than $T_{\text {sat }}+4 \mathrm{~K}$, where $T_{\text {sat }}$ is here the saturation temperature in the groove. The inlet pressure is $P_{c c}=615050 \mathrm{~Pa}$, which corresponds to the saturation temperature $T_{\text {sat }}=283.15 \mathrm{~K}$. A grid of $31 \times 49 \times 33$ points (leading a total number of 50,127 computational points) is used. The grid has $31 \times 20 \times 33$ points (which corresponds to 20,460 computational points) located within the wick and $20 \times 13 \times 33+31 \times 16 \times 33$ (which corresponds to 16,368 computational points) located within the casing.

As indicated in Table 3, the bond diameters are in the range 1$3 \mu \mathrm{m}$. By analogy with a random packing of spheres, the mean distance between two pores ( $\sim$ sphere diameter) can be expected to be on the order of $10 \mu \mathrm{m}$. According to the wick unit cell dimensions reported in Table 3, such a value of the lattice spacing would leads to consider a $186 \times 120 \times 200$ network for representing the wick. Considering such a network is not possible in practice with the present version of the code because of the too long corresponding computational times. The simulations presented hereafter were performed with a greater lattice spacing ( $a=61 \mu \mathrm{m})$ so as to obtain reasonable computational times. The average computational time for a given heat load with two-phase zone formation for the grid with $31 \times 49 \times 33$ points is about $1 \mathrm{~h}$ on a PC. To obtain the results discussed below, the imposed heat flux was varied in the range [0.8-12 W/cm $\left./ \mathrm{cm}^{2}\right]$ by increment of $0.005 \mathrm{~W} / \mathrm{cm}^{2}$, which leads to 2240 simulations and about 90 days of computational time. 
Table 2

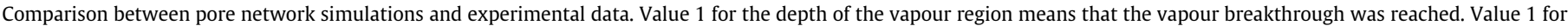
occupancy ratio of a groove-wick interface means that the all the pores along the considered interface are occupied by vapour.

\begin{tabular}{|c|c|c|c|c|}
\hline Simulation & Depth of the vapour & Left groove-wick interface occupancy & Right groove-wick interface occupancy & Vapour saturation \\
\hline 1 & 1 & 0.32 & 0.24 & 0.51 \\
\hline 2 & 0.92 & 1 & 1 & 0.63 \\
\hline 3 & 0.86 & 0.55 & 0.43 & 0.51 \\
\hline 4 & 1 & 0.55 & 0.52 & 0.62 \\
\hline 5 & 0.97 & 0.48 & 0.81 & 0.57 \\
\hline 6 & 1 & 0.45 & 0.33 & 0.55 \\
\hline 7 & 0.79 & 1 & 1 & 0.57 \\
\hline 8 & 0.88 & 0.55 & 0.43 & 0.54 \\
\hline 9 & 0.99 & 1 & 1 & 0.63 \\
\hline 10 & 0.86 & 0.27 & 0.52 & 0.52 \\
\hline 11 & 0.90 & 0.82 & 0.86 & 0.64 \\
\hline 12 & 1 & 0.32 & 0.71 & 0.63 \\
\hline 13 & 0.86 & 0.50 & 0.43 & 0.56 \\
\hline Average & 0.92 & 0.62 & 0.61 & 0.57 \\
\hline Standard deviation/average & 0.08 & 0.43 & 0.44 & 0.08 \\
\hline Experimental data & 1 & 0.5 & 0.75 & 0.55 \\
\hline
\end{tabular}
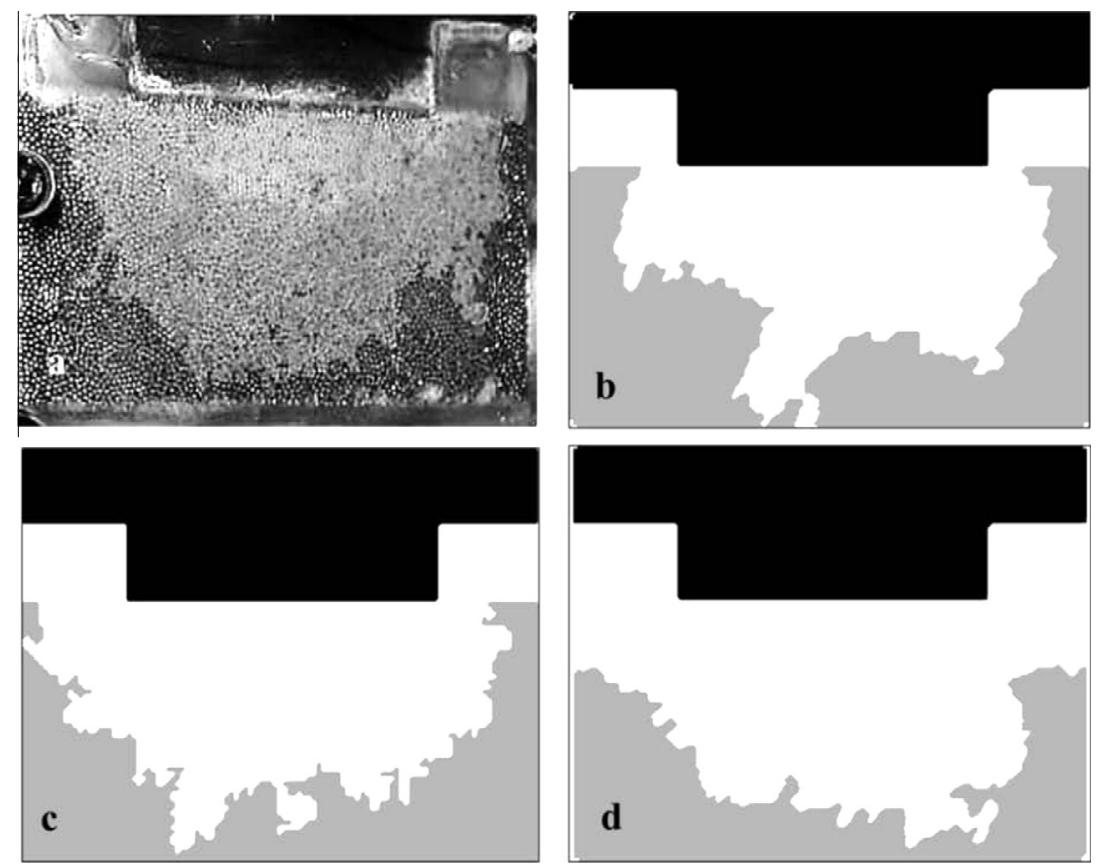

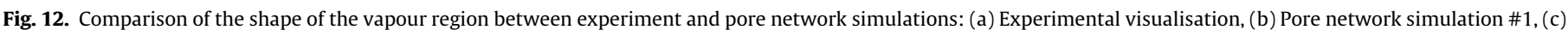
Pore network simulation \# 5 and (d) Pore network simulation \#9.

A series of steady-state solutions is thus obtained by varying the heat flux applied on the casing outer surface. As in previous works, e.g. $[14,18]$, the nucleation temperature is not reached in the wick for sufficiently low heat flux. Vapourization takes place only at the wick-groove interface and the wick operates in a liquid saturated mode. For higher heat fluxes, partial invasion of the wick by the vapour occurs. This was also predicted in the aforementioned previous works. However, as announced in the introduction section, the 3D simulations lead to identify a regime, referred to as regime II, which is fundamentally different from the regimes reported in [14] or [18]. This regime is obtained for an intermediate range of heat fluxes, sufficiently high for the wick to be partially invaded by the vapour but not too high as discussed hereafter. As illustrated in Fig. 14b, this regime is characterised by the coexistence of the liquid and vapour phases over the casing-wick contact surface. This is in contrast with the results reported in [14] or [18], where only vapour is in contact with the casing as soon as the wick becomes unsaturated.

More details on the variation of phase distribution with heat flux are presented in Fig. 15. This figure shows the fraction of pores right under the casing occupied by either vapour or liquid, the overall vapour saturation in the wick and the depth of the two-phase region, which is the distance between the casing wick interface and the pore occupied by vapour located the closest to the wick inlet, as a function of heat flux applied on the casing.

As can be seen, regime II, where a two-phase zone is in contact with the casing, extends over a significant range of heat fluxes. The fraction of liquid pores in contact with the casing exceeds the fraction of vapour pores in contact with the casing up to an applied heat flux of $2.5 \mathrm{~W} / \mathrm{cm}^{2}$. For this flux, the depth of the two-phase region is already high and reaches $67 \%$ of the wick thickness even though only $10 \%$ of the wick pore space is occupied by vapour. The 


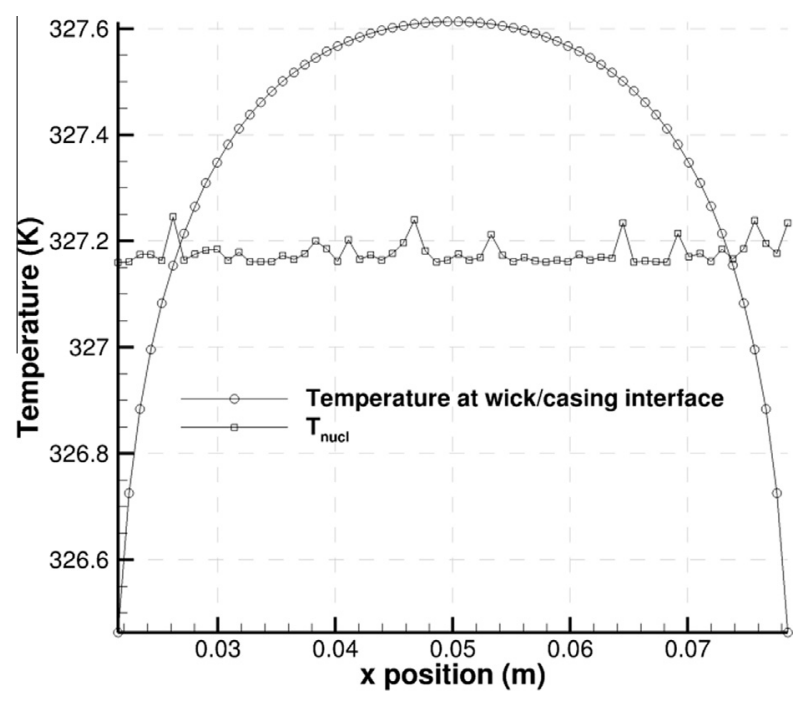

Fig. 13. Computed distribution of temperature along the casing-wick interface for an applied flux of $0.5 \mathrm{~W} / \mathrm{m}^{2}$.

wick/casing surface becomes fully dry for heat fluxes greater than $10 \mathrm{~W} / \mathrm{cm}^{2}$. The vapour saturation increases rapidly with the applied heat load in this regime but remains relatively low (below $17 \%)$. This is in sharp contrast with the $2 \mathrm{D}$ simulation results reported in which led to much higher vapour saturations for similar applied heat fluxes. The overall vapour saturation seems to stabilize in Fig. 15. Although not shown here, the vapour saturation continues to increase with the heat flux but less rapidly once a dry vapour region is present under the casing and eventually forms a thick vapour blanket. As can be seen from Fig. 15, the depth of the two-phase region within the wick reaches a plateau in regime II. This plateau corresponds to the growth of the two-phase region in the lateral direction in the wick instead of the depth direction.

In summary the 3D simulations show the existence of three regimes and not only two as reported in some previous investigations, e.g. [14] or [18]: a regime at low heat load where the wick is

Table 3

Porous materials properties, fluid (ammonia) properties and geometrical dimensions used for the 3D simulations.

\begin{tabular}{|c|c|c|}
\hline \multicolumn{3}{|l|}{ Wick properties } \\
\hline Porosity & \multicolumn{2}{|l|}{$\varepsilon=0.59$} \\
\hline Permeability & \multicolumn{2}{|l|}{$K=43.10^{-14} \mathrm{~m}^{2}$} \\
\hline $\begin{array}{l}\text { Solid phase thermal } \\
\text { conductivity }\end{array}$ & \multicolumn{2}{|l|}{$\lambda_{w}=4.18 \mathrm{~W} / \mathrm{m} / \mathrm{K}$} \\
\hline Bond diameter range & \multicolumn{2}{|l|}{$1-3 \mu \mathrm{m}$} \\
\hline Bond diameter p.d.f. & \multicolumn{2}{|l|}{ Uniform } \\
\hline \multicolumn{3}{|l|}{ Casing property } \\
\hline Thermal conductivity & \multicolumn{2}{|l|}{$\lambda_{c}=70 \mathrm{~W} / \mathrm{m} / \mathrm{K}$} \\
\hline \multicolumn{3}{|l|}{ Fluid properties } \\
\hline & Liquid & Vapor \\
\hline Thermal conductivity & $\lambda_{l}=0.52912 \mathrm{~W} / \mathrm{m} / \mathrm{K}$ & $\lambda_{v}=0.024365 \mathrm{~W} / \mathrm{m} / \mathrm{K}$ \\
\hline Dynamic viscosity & $\mu_{l}=156.10^{-6}$ Pa.s & $\mu_{v}=9,63 \cdot 10^{-6}$ Pa.s \\
\hline Specific heat capacity & $c_{p, l}=4675.7 \mathrm{~J} / \mathrm{kg} / \mathrm{K}$ & $c_{p, v}=2841.3 \mathrm{~J} / \mathrm{kg} / \mathrm{K}$ \\
\hline Latent heat of vaporization & $h_{l v}=1225580 \mathrm{~J} / \mathrm{kg}$ & \\
\hline Interfacial tension & $\sigma=0.029589 \mathrm{~N} / \mathrm{m}$ & \\
\hline Liquid density & $\rho_{l}=624.46 \mathrm{~kg} / \mathrm{m}^{3}$ & \\
\hline \multicolumn{3}{|c|}{ Geometrical dimensions ( $\mathrm{mm}$ ) (see Fig. 2 for definitions) } \\
\hline$L_{x}$ & \multicolumn{2}{|l|}{1.9} \\
\hline$L_{y}$ & \multicolumn{2}{|l|}{3} \\
\hline$L_{z}$ & \multicolumn{2}{|l|}{2} \\
\hline$L_{x v}$ & \multicolumn{2}{|l|}{0.35} \\
\hline$L_{y w}$ & \multicolumn{2}{|l|}{1.2} \\
\hline$L_{y g}$ & \multicolumn{2}{|l|}{0.8} \\
\hline
\end{tabular}

saturated by liquid, a regime at high heat load where the wick-casing surface is dry and an intermediate regime (regime II) where both liquid and vapour are in contact with the casing-wick surface. These three regimes are illustrated in Fig. 14.

\subsection{Vapourization partition}

The mass flow rate of vapour feeding the vapour groove in regime II is shown as a function of the heat flux applied on the casing in Fig. 16. The mass flow rate is divided in two parts: the fraction of the total mass flow rate resulting from vapourization of the liquid at the wick/groove interface and the fraction of the total mass flow rate resulting from vapourization within the wick. As can be seen the vapour which feeds the groove mostly comes from the vapourization process within the wick in regime II.

Fig. 16 also shows a significant increase, from $32.7 \%$ to $52.6 \%$, in the fraction of vapour mass flow rate resulting from the vapourization process within the wick at the beginning of regime II when the heat flux varies from $1.38 \mathrm{~W} / \mathrm{cm}^{2}$ to $1.385 \mathrm{~W} / \mathrm{cm}^{2}$. This corresponds to the situation where the two-phase region becomes connected to the two grooves on both sides of casing fin. For lower heat loads in regime II, the two-phase zone is connected to only one groove.

\subsection{Maximum temperature}

The maximum temperature $T_{\max }$ in our computational domain is located at the metallic casing outer surface (at $y=L_{y}$, see Fig. 2). The temperature is actually quasi-uniform along the casing outer surface, only very slightly greater on the left and right of casing outer surface. In practice, this temperature should not exceed some specified value depending on the equipment to be cooled. The maximum temperature can be characterised from the excess temperature $\Delta T_{\max }=T_{\max }-T_{\text {sat }}$, where $T_{\text {sat }}$ is the saturation temperature inside the grooves. As shown in Fig. 17, the excess temperature $\Delta T_{\max }$ is equal to about $26 \mathrm{~K}$ for a heat load of $11.9 \mathrm{~W} /$ $\mathrm{cm}^{2}$, i.e. at the end of regime II. This order of magnitude is quite acceptable for such a system, indicating that regime II is a fully operational regime, i.e. is quite likely in operating LHP. The variation of $\Delta T_{\max }$ with the heat flux is not perfectly monotonous in Fig. 17. This is due to the discrete representation of pore space. A slight decrease in $\Delta T_{\max }$ can happen when a new pore is occupied by vapour at the wick-groove interface.

\subsection{Conductance}

The global performance of evaporator can be characterised from the computation of its global conductance defined as:

$C_{\text {evap }}=\frac{Q}{T_{\max }-T_{g}}$

where $T_{g}$ is the temperature in the groove $\left(T_{g}=T_{s a t}\left(P_{g}\right)\right)$. The variation of $C_{\text {evap }}$ with the applied heat flux $Q$ is shown in Fig. 18. The results are markedly different from those obtained from 2D PN simulations, [18], which indicated that the conductance was maximum in regime I (fully saturated wick) and then decreased as soon as the vapour penetrated in the wick. The 3D simulations reveal that the evaporator conductance is the greatest in regime II, i.e. with a partially saturated wick. Actually, three different ranges of flux can be distinguished in Fig. 18.

The conductance, which is constant in regime I (liquid saturated wick), increases in regime II with the heat load until reaching a maximum (on the order of $0.57 \mathrm{~W} / \mathrm{cm}^{2} / \mathrm{K}$ in our simulations). Note that this maximum is observed over a quite significant range of heat flux in regime II (as shown by the plateau in Fig. 18). The 


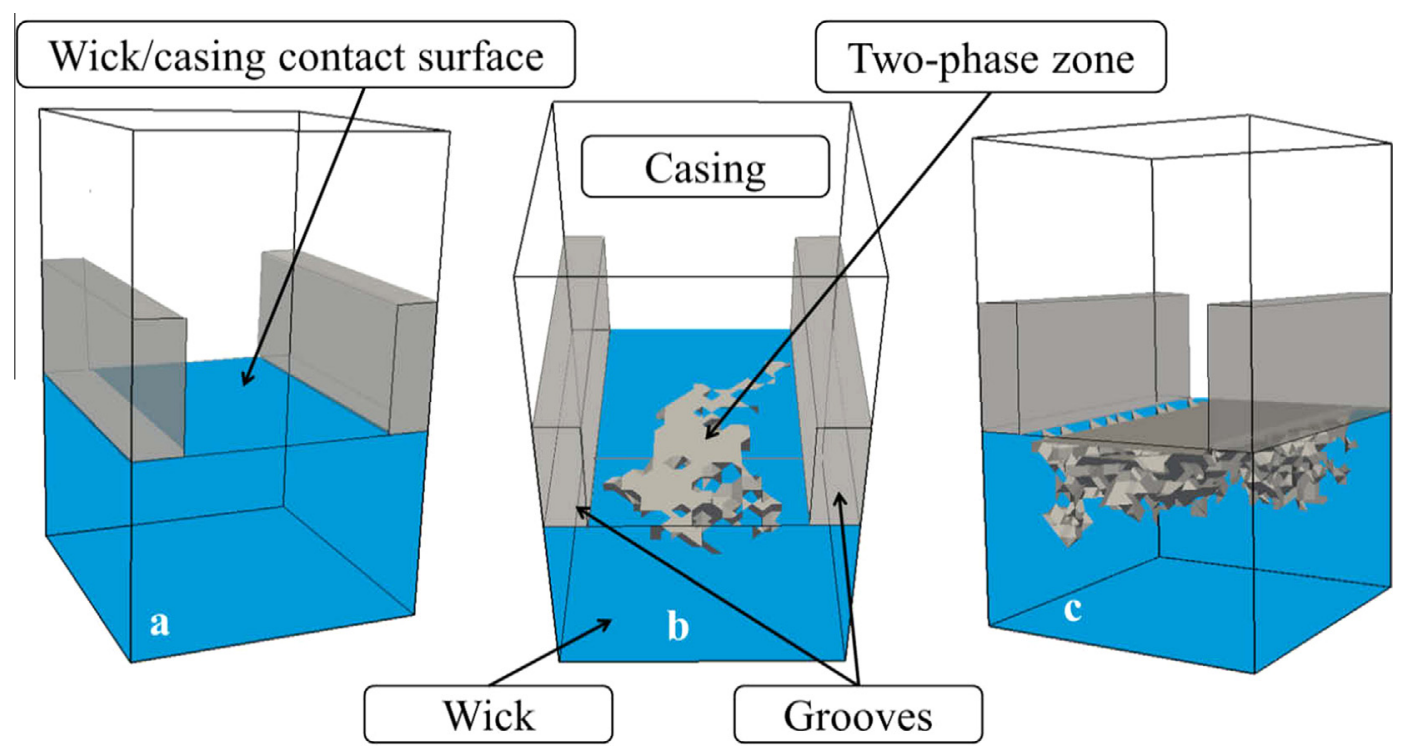

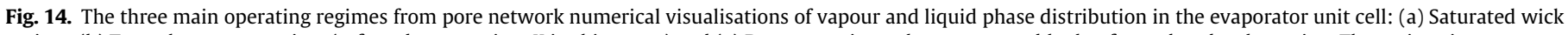

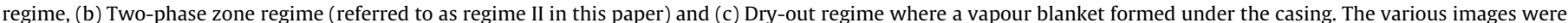

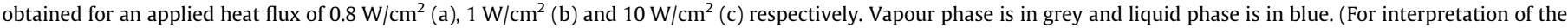
references to colour in this figure legend, the reader is referred to the web version of this article.)

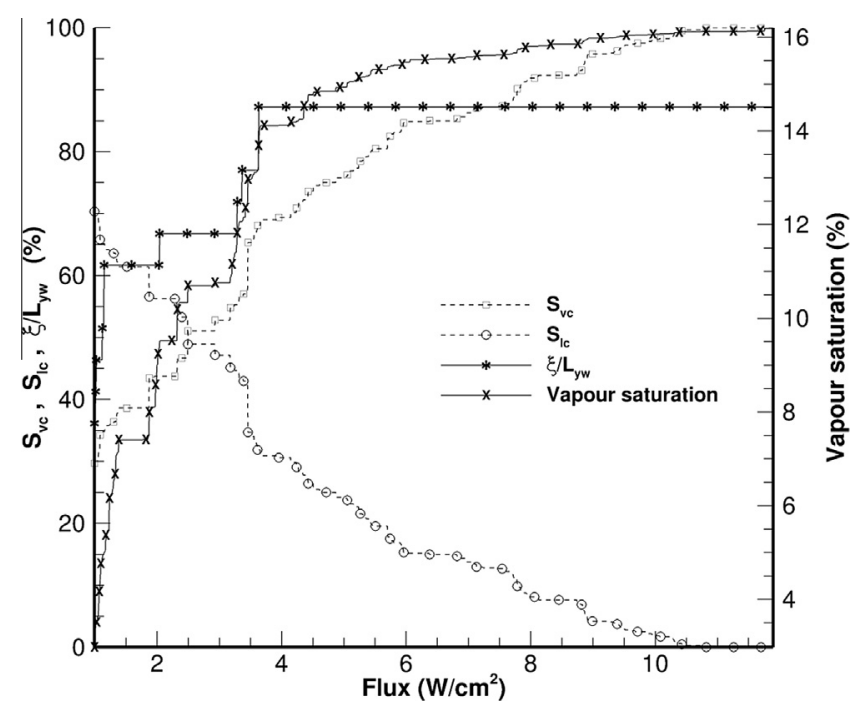

Fig. 15. Variation of vapour region depth (divided by wick thickness), vapour overall saturation and the occupancy state of pores under the casing as a function of heat flux applied on the casing. $S_{v c}$ is the fraction of pore occupied by vapour right under the casing, $S_{l c}$ is the fraction of pore occupied by liquid right under the casing and $\xi / L_{y w}$ is the depth of the two phase zone.

conductance decreases in regime II when the fraction of the casing-wick contact surface in contact with liquid becomes too small. In our simulations, the conductance begins to decrease for an applied heat flux of about $7.8 \mathrm{~W} / \mathrm{cm}^{2}$, that is when less than about $10 \%$ of the pores in contact with the casing are still occupied by liquid (see Fig. 15). From the results reported in Figs. 15 and 18, the best evaporator performance is observed in the range of heat fluxes [3.7-7.8 W/ $/ \mathrm{cm}^{2}$ ] corresponding to a noticeable fraction of pores in contact with the casing occupied by liquid. This fraction varies between $35 \%$ and $10 \%$ in this range of heat fluxes according to Fig. 15.

Interestingly, the conductance variation shown in Fig. 18 is qualitatively similar to the variation obtained in the experiment of Zhao and Liao [19], see Fig. 4 in [19].

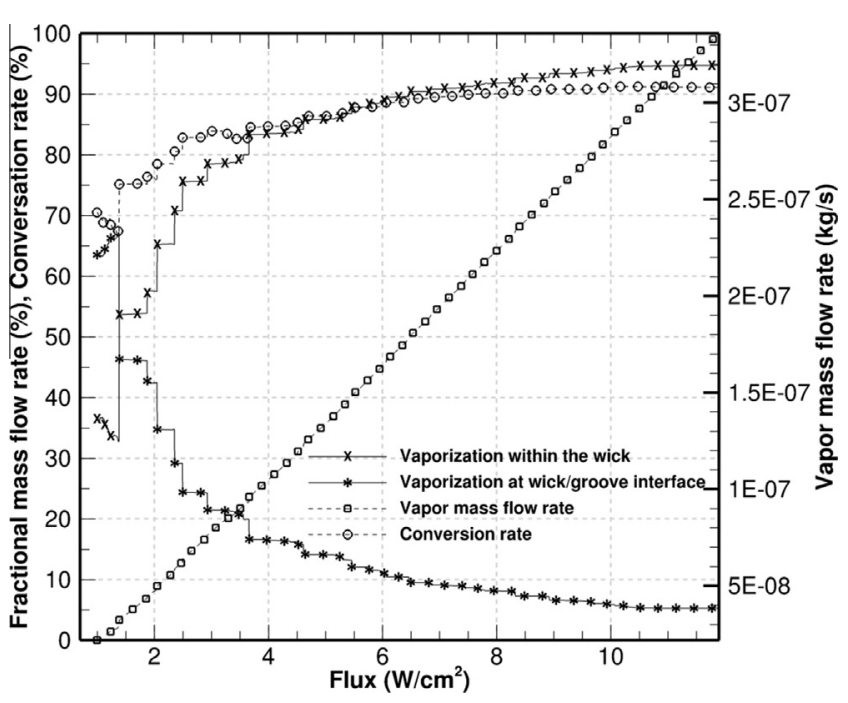

Fig. 16. Variation of vapour mass flow rate partition, vapour mass flow rate and conversion rate of applied flux as a function of the heat flux applied on the casing in regime II.

The theory of invasion percolation in a gradient, see [22] and references therein, indicates that the size of two-phase phase, which roughly correspond to the depth $\xi$ of two phase-zone region should scale as

$\frac{\xi}{a} \propto\left(\frac{C a}{\Sigma}\right)^{-1}$

where the capillary number $\mathrm{Ca}$ can be expressed as $C a=\mu_{l} Q /\left(h_{l v} L_{x} L_{z} \sigma\right)$ and $\Sigma=\left(d_{\max }-d_{\min }\right) / a$ (where it is recalled that $a$ is the lattice spacing, which can be seen as the mean distance between two pores). Eq. (29) thus indicates that a broad pore size distribution (i.e. a relatively large $\Sigma$ ) favours the formation of a two-phase zone while a narrow distribution will favour the detrimental formation of a vapour blanket under the casing. In 


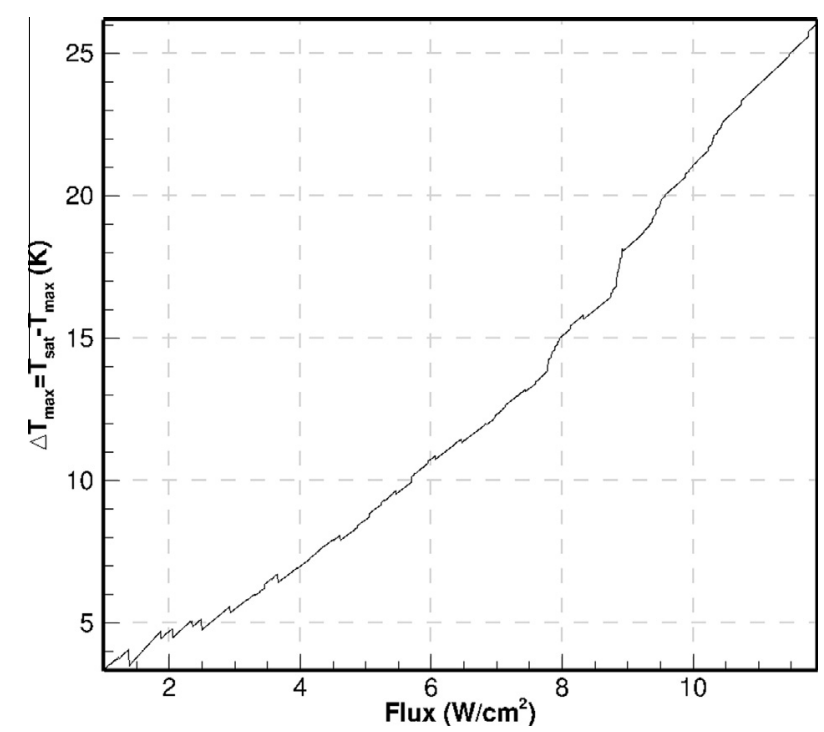

Fig. 17. Variation of $\Delta T_{\max }=T_{\max }-T_{\text {sat }}$ as a function of the heat load applied on the casing in regime II. $T_{\text {sat }}$ is the saturation temperature inside the grooves.

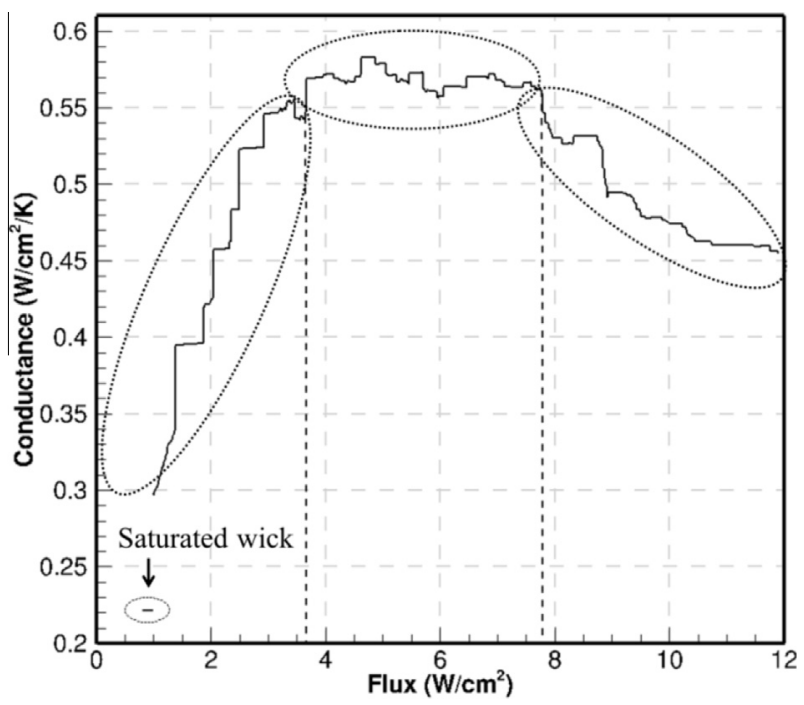

Fig. 18. Variation of evaporator conductance as a function of the heat load applied on the casing.

our simulations, the pore size distribution is narrow. Therefore, the occurrence of regime II in a capillary evaporator is actually still more likely than predicted from our simulations.

\section{Conclusions}

Simulations of heat and mass transfer with phase change in a capillary evaporator representative unit cell were presented in this paper. The simulations are based on a mesoscale approach in which the capillary effects are modelled as in classical pore network models but where the pressure and temperature fields are computed using mean field approaches. This mesoscale model, referred to as a mixed pore network model, was first qualitatively validated by comparison with a visualisation experiment using a quasi-two dimensional model porous wick.

This experiment indicated that nucleation could occur under the casing at some distance from the grooves as well as near the triple contact line regions between the wick, the casing and the groove. This feature was also in reasonable agreement with the simulations and the rules adopted in the model as regards the vapour nucleation in the wick. Also, it can be noted that the number of vapour growth incipient sites in the wick or at its surface was quite low in this experiment, typically only 1 or 2 , which is also in accordance with the assumption made in the model Although essentially qualitative, the satisfactory agreement between the simulations and this experiment enables one to use with some confidence the mixed pore network model to study the heat and mass transfer in the wick of a capillary evaporator.

The 3D simulations indicate that three regimes should be distinguished as regards the distribution of the fluid within the wick when the applied heat load is varied. This is in contrast with several previous works where only two regimes were distinguished. In particular, the 3D simulation indicates that the regime leading to the best evaporator performance is a regime, referred to as regime II, where a two-phase liquid-vapour zone forms within the wick. In this regime, the liquid phase in the wick is in partial contact with the casing at the casing/wick contact surface. Because of the fundamental differences between the percolation properties of the liquid and vapour phases in the wick between the 2D case and the 3D case, this regime was completely overlooked in some previous works based on 2D pore network simulations. In particular, this regime could not be studied in the previous modelling approaches assuming only two regions in the wick separated by a macroscopic sharp interface: a dry region occupied by vapour and a liquid region only occupied by liquid. The 3D simulations indicate that three types of region can in fact exist in the wick (in regime III, i.e. for sufficiently high heat load): dry regions, liquid saturated regions and two-phase regions where vapour and liquid coexist. However, the formation of a dry region along the casing in regime III leads to a significant degradation in the evaporator performance.

Therefore, the 3D simulations suggest that the porous wick should be designed so as to favour the occurrence of regime II over a large range of heat loads.

\section{Conflict of interest}

None declared.

\section{Acknowledgements}

Financial supports from CNES and Airbus Defence and Space are gratefully acknowledged.

\section{Appendix A. Convective heat transfer coefficient in the grooves}

A convective boundary condition is used at the casing-groove interface and wick-groove interface when the pore is occupied by vapour as expressed by Eqs. (15) and (20).

The heat convective coefficient $h_{c}$ is computed using the correlation given by Sleicher and Rouse [26]. This correlation is expressed in terms of the Nusselt number and is valid for a fully developed flow inside a rectangular pipe:

$N u=\frac{h_{c} D_{H}}{\lambda_{v}}=5+0.015 \operatorname{Re}^{a} \operatorname{Pr}^{b}$

with $a=0.88-0.24 / 4+\operatorname{Pr} \quad$ and $\quad b=\frac{1}{3}+0.5 \exp (-0.6 \operatorname{Pr})$. The Reynolds number and the Prandlt number are determined as $\operatorname{Re}=\bar{u}_{v} \rho_{v} D_{H} / \mu_{v}$ and $\operatorname{Pr}=c_{p, v} \mu_{v} / k_{v}$.

\section{Appendix B. Computation of pressure drop inside the loop}

The pore network model is coupled with a loop model in order to determine the pressure in the grooves. The loop model is based 
on the model presented in [9]. Values of the loop model parameters are taken from [29]. Note, however, that the saturation pressure inside the compensation chamber is fixed in our simulations whereas this is an output in Li and Peterson approach. We recall that the following elements were considered between the exit of the groove and the liquid chamber: the vapour line, the condenser, the liquid line, denoted by the subscript $v l$, cond and $l l$ respectively.

The mass flow rate entering the wick is expressed as

$\dot{m}_{l}=\rho_{l} u_{l} S$

where $S=L_{x} \times L_{z}$ is the wick inlet area. Note that $\dot{m}_{v}=\dot{m}_{l}$ in the vapour line since the mass flow rate is conserved all along the loop in the steady-state regime. The pressure losses in the loop are balanced by the action of capillary forces:

$\Delta P_{\text {cap }}=\Delta P_{v}+\Delta P_{l}+\Delta P_{w}$

where the pressure losses in the wick and in the liquid and vapour sections of the loop are decomposed into:

$\Delta P_{l}=\Delta P_{l l}+\Delta P_{l, \text { cond }}$

$\Delta P_{v}=\Delta P_{g}+\Delta P_{v l}+\Delta P_{v, \text { cond }}$

$\Delta P_{w}=P_{c c}-P_{l, w}$

while the capillary pressure is given by

$\Delta P_{\text {cap }}=P_{g}-P_{l, w}$

The goal is to determine the vapour pressure $P_{g}$ inside the groove. Combining Eqs. (B2)-(B6), the vapour pressure inside the grooves can be expressed as follows:

$P_{g}=P_{c c}+\Delta P_{v}+\Delta P_{l}$

Assuming a circular pipe of diameter $d_{l l}$, the pressure drop inside the liquid line is determined as follows:

$\Delta P_{l l}=\frac{128 l_{l l} v_{v} \dot{m}_{l}}{\pi d_{l l}^{4}}$

Similarly, the pressure drop inside the vapour line of diameter $d_{v l}$ is expressed as

$\Delta P_{v l}=\frac{128 l_{v l} v_{v} \dot{m}_{v}}{\pi d_{v l}^{4}}$

The pressure drop inside the condenser is computed as the sum of two pressure drops, still using the Poseuille's law in a circular pipe of diameter $d_{\text {cond }}$,

$\Delta P_{v, \text { cond }}=\frac{128 l_{v, \text { cond }} v_{v} \dot{m}_{v}}{\pi d_{\text {cond }}^{4}}$

$\Delta P_{l, \text { cond }}=\frac{128 l_{l, \text { cond }} v_{l} \dot{m}_{l}}{\pi d_{\text {cond }}^{4}}$

The pressure drop in the vapour inside the groove is computed as explained in the works of $\mathrm{Li}$ and Peterson [30,31,9]:

$\Delta P_{v, \text { groove }}=2 \frac{\rho_{\nu} v_{v} \gamma \overline{\bar{u}_{v}}}{D_{H}^{2}} L_{z}$

where $D_{H}=4 S / P=\left(8 L_{x v} L_{y g}\right) /\left(4 L_{x v}+2 L_{y g}\right)$ is the hydraulic diameter, $\bar{u}_{v}=\dot{m}_{v} /\left(2 N L_{x v} L_{y g} \rho_{v}\right)$ is the mean velocity of vapour with $N$ the number of grooves, $\gamma=4.7+19.64 G$ with $G=\left(A^{2}+1\right) /(A+1)^{2}$ and $A=L_{y g} /\left(2 L_{x v}\right)$.

\section{References}

[1] J. Ku, Operating characteristics of loop heat pipes, in: SAE Technical Report, Proceeding of the 29th International Conference on Environmental System, Denver, USA, 1999.
[2] Y.F. Maydanik, Loop heat pipes, Appl. Therm. Eng. 25 (5) (2005) 635-657.

[3] S. Launay, V. Sartre, J. Bonjour, Parametric analysis of loop heat pipe operation: a literature review, Int. J. Therm. Sci. 46 (7) (2007) 621-636.

[4] T. Kaya, R. Pérez, C. Gregori, A. Torres, Numerical simulation of transient operation of loop heat pipes, Appl. Therm. Eng. 28 (8) (2008) 967-974.

[5] B. Siedel, V. Sartre, F. Lefèvre, Numerical investigation of the thermohydraulic behaviour of a complete loop heat pipe, Appl. Therm. Eng. 61 (2) (2013) 541 553.

[6] M.A. Chernysheva, Y.F. Maydanik, Numerical simulation of transient heat and mass transfer in a cylindrical evaporator of a loop heat pipe, Int. J. Heat Mass Transfer 51 (17) (2008) 4204-4215.

[7] M.A. Chernysheva, Y.F. Maydanik, 3D-model for heat and mass transfer simulation in flat evaporator of copper-water loop heat pipe, Appl. Therm. Eng. 34 (2012) 124-134.

[8] Y. Cao, A. Faghri, Analytical solutions of flow and heat transfer in a porous structure with partial heating and evaporation on the upper surface, Int. J. Heat Mass Transfer 37 (10) (1994) 1525-1533.

[9] J. Li, G. Peterson, 3D heat transfer analysis in a loop heat pipe evaporator with a fully saturated wick, Int. J. Heat Mass Transfer 54 (1) (2011) 564-574.

[10] Y. Xuan, K. Zhao, Q. Li, Investigation on heat and mass transfer in a evaporator of a capillary-pumped loop with the lattice Boltzmann method: pore scale simulation, Transp. Porous Media 89 (3) (2011) 337-355.

[11] Z. Wan, J. Liu, J. Wan, Z. Tu, W. Lu, An overall numerical investigation on heat and mass transfer for miniature flat plate capillary pumped loop evaporator, Thermochim. Acta 518 (1) (2011) 82-88.

[12] X. Zhang, X. Li, S. Wang, Three-dimensional simulation on heat transfer in the flat evaporator of miniature loop heat pipe, Int. J. Therm. Sci. 54 (2012) 188198.

[13] A.S. Demidov, E.S. Yatsenko, Investigation of heat and mass transfer in the evaporation zone of a heat pipe operating by the 'inverted meniscus' principle, Int. J. Heat Mass Transfer 37 (14) (1994) 2155-2163.

[14] C. Figus, Y. Le Bray, S. Bories, M. Prat, Heat and mass transfer with phase change in a porous structure partially heated: continuum model and pore network simulations, Int. J. Heat Mass Transfer 42 (14) (1999) 2557-2569.

[15] T. Kaya, J. Goldak, Numerical analysis of heat and mass transfer in the capillary structure of a loop heat pipe, Int. J. Heat Mass Transfer 49 (17) (2006) 3211 3220.

[16] Z.C. Liu, W. Liu, A. Nakayama, Flow and heat transfer analysis in porous wick of CPL evaporator based on field synergy principle, Heat Mass Transfer 43 (12) (2007) 1273-1281.

[17] C. Ren, Q.-S. Wu, M.-B. Hu, Heat transfer with flow and evaporation in loop heat pipe's wick at low or moderate heat fluxes, Int. J. Heat Mass Transfer 50 (11) (2007) 2296-2308.

[18] T. Coquard, M. Prat, A. Larue de Tournemine, C. Figus, Pore network models as a tool for the analysis of heat and mass transfer with phase change in the capillary structure of loop heat pipe, in: Proceeding of the 14th International Heat Pipe Conference, Florianopolis, Brazil, 2007.

[19] T.S. Zhao, Q. Liao, On capillary-driven flow and phase-change heat transfer in a porous structure heated by a finned surface: measurements and modeling, Int. J. Heat Mass Transfer 43 (7) (2000) 1141-1155.

[20] Y.H. Yan, J. Ochterbeck, Numerical investigation of the steady-state operation of a cylindrical capillary pumped loop evaporator, J. Electron. Packag. 125 (2) (2003) 251-260.

[21] M. Prat, Application of pore network models for the analysis of heat and mass transfer with phase in the porous wick of loop heat pipes, Heat Pipe Sci. Technol. 1 (2) (2010) 129-149.

[22] M. Prat, S. Veran-Tissoires, N. Vorhauer, T. Metzger, E. Tsotsas, Fractal phase distribution and drying: impact on two-phase zone scaling and drying time scale dependence, Drying Technol. 30 (11) (2012) 1129-1135.

[23] M.J. Blunt, M.D. Jackson, M. Piri, P.H. Valvatne, Detailed physics, predictive capabilities and macroscopic consequences for pore-network models of multiphase flow, Adv. Water Resour. 25 (8) (2002) 1069-1089.

[24] J. Bear et, Y. Bachmat, Introduction to Modeling of Transport Phenomena in Porous Media, Kluwer Academic Publishers, Dordrecht, 1990.

[25] S.V. Patankar, Numerical Heat Transfer and Fluid Flow, Hemisphere Publishing Corporation, USA, 1980, pp. 10-22.

[26] C.A. Sleicher, M. Rouse, A convenient correlation for heat transfer to constant and variable property fluids in turbulent pipe flow, Int. J. Heat Mass Transfer 18 (5) (1975) 677-683.

[27] D. Wilkinson, J.F. Willemsen, Invasion percolation: a new form of percolation theory, J. Phys. A: Math. Gen. 16 (14) (1983) 3365-3376.

[28] T. Coquard, Transferts couplés de masse et de chaleur dans un élément d'évaporateur capillaire (Ph.D. thesis), Institut National Polytechnique de Toulouse, Toulouse, France, 2006.

[29] J. Li, D. Wang, G. Peterson, A compact loop heat pipe with flat square evaporator for high power chip cooling, IEEE Trans. Compon. Packag. Technol. 1 (4) (2011) 519-527.

[30] J. Li, G. Peterson, Geometric optimization of a micro heat sink with liquid flow, IEEE Trans. Compon. Packag. Technol. 29 (1) (2006) 145-154.

[31] J. Li, G. Peterson, 3-Dimensional numerical optimization of silicon-based high performance parallel microchannel heat sink with liquid flow, Int. J. Heat Mass Transfer 50 (15) (2007) 2895-2904. 